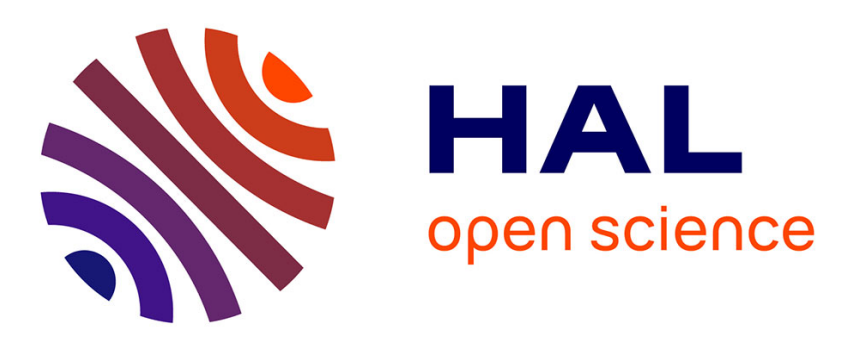

\title{
Ru-Catalyzed Selective C-H Bond Hydroxylation of Cyclic Imides
}

Yu-Chao Yuan, Christian Bruneau, Vincent Dorcet, Thierry Roisnel, Rafael Gramage-Doria

\section{- To cite this version:}

Yu-Chao Yuan, Christian Bruneau, Vincent Dorcet, Thierry Roisnel, Rafael Gramage-Doria. RuCatalyzed Selective C-H Bond Hydroxylation of Cyclic Imides. Journal of Organic Chemistry, 2019, 84 (4), pp.1898-1907. 10.1021/acs.joc.8b02899 . hal-02020140

\section{HAL Id: hal-02020140 https://hal-univ-rennes1.archives-ouvertes.fr/hal-02020140}

Submitted on 16 May 2019

HAL is a multi-disciplinary open access archive for the deposit and dissemination of scientific research documents, whether they are published or not. The documents may come from teaching and research institutions in France or abroad, or from public or private research centers.
L'archive ouverte pluridisciplinaire HAL, est destinée au dépôt et à la diffusion de documents scientifiques de niveau recherche, publiés ou non, émanant des établissements d'enseignement et de recherche français ou étrangers, des laboratoires publics ou privés. 


\title{
Ru-Catalyzed Selective C-H Bond Hydroxylation of Cyclic Im- ides
}

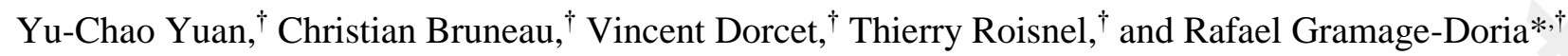 \\ Univ Rennes, CNRS, ISCR - UMR 6226, F-35000 Rennes, France
}

\section{Supporting Information Placeholder}

\begin{abstract}
We report on cyclic imides as weak directing groups for selective mono-hydroxylation reactions using ruthenium catalysis. Whereas acyclic amides are known to promote the hydroxylation of the $\mathrm{C}\left(\mathrm{sp}^{2}\right)-\mathrm{H}$ bond enabling 5-membered ring ruthenacycle intermediates, the cyclic imides studied herein enabled the hydroxylation of the $\mathrm{C}\left(\mathrm{sp}^{2}\right)$ - $\mathrm{H}$ bond via a larger 6-membered ruthenacycle intermediates. Furthermore, mono-hydroxylated products were exclusively obtained (even in the presence of over-stoichiometric amounts of reagents), which was rationalized by the difficulty to accommodate co-planar intermediates once the first hydroxyl group was introduced into the substrate. The same reactivity was observed in the presence of palladium catalysts.
\end{abstract}

\section{INTRODUCTION}

Catalytic oxidations have become increasingly important in the last decades with the aim of replacing toxic and hazardous reagents in industrial and academic laboratories. ${ }^{1}$ Additionally, they provide a plethora possibilities to achieve selectivity patterns that are per se difficult or impossible to tackle with non-catalyzed reactions that traditionally require harsher reaction conditions than the catalytic ones. ${ }^{2}$ In this context, particular focus has been devoted to catalytic oxidations such as hydroxylation, epoxidation, acetoxylation and benzoxylation of $\mathrm{C}-\mathrm{H}$ bonds sought to be traditionally unreactive. ${ }^{3}$ Bio-inspired iron, copper and manganese complexes are the prevalent catalysts for aliphatic and alkene $\mathrm{C}-\mathrm{H}$ bond oxidations as they promote oxygen atom transfer mechanisms via key oxo species. ${ }^{4}$ On the other hand, the aromatic $\mathrm{C}-\mathrm{H}$ bond oxidations are usually accomplished with palladium and ruthenium catalysts that enable the key C-H bond activation step via a key metallacycle intermediate assisted by a directing group. ${ }^{5}$ In the former case, most of the examples have been performed with substrates containing a single aromatic fragment, which significantly decreases and simplifies the number of the products that can be formed. ${ }^{6}$ Indeed, in the case where two aromatic fragments are present around the same directing group, undesired regio-selectivity issues as well as over-oxidation reactions are encountered. ${ }^{7}$ An interesting strategy to circumvent this problem was reported by Rao and co-workers who demonstrated the regio-divergent catalytic behavior of ruthenium and palladium towards flexible, acyclic tertiary amides containing two aromatic fragments (Scheme 1A). ${ }^{8}$ Whereas the ruthenium catalyst formed the 5-membered metallacycle intermediate enabling the hydroxylation at the ortho $\mathrm{C}-\mathrm{H}_{\mathrm{a}}$ bond of the phenyl ring A attached to the carbonyl group, the palladium catalyst favored the 6-membered metallacycle intermediate that promoted the hydroxylation at the ortho $\mathrm{C}-\mathrm{H}_{\mathrm{b}}$ bond of the phenyl ring $\mathrm{B}$ attached to the nitrogen atom.
The carbonyl group behaved in both cases as a weak directing group for this type of flexible substrates. ${ }^{8}$

Scheme 1.

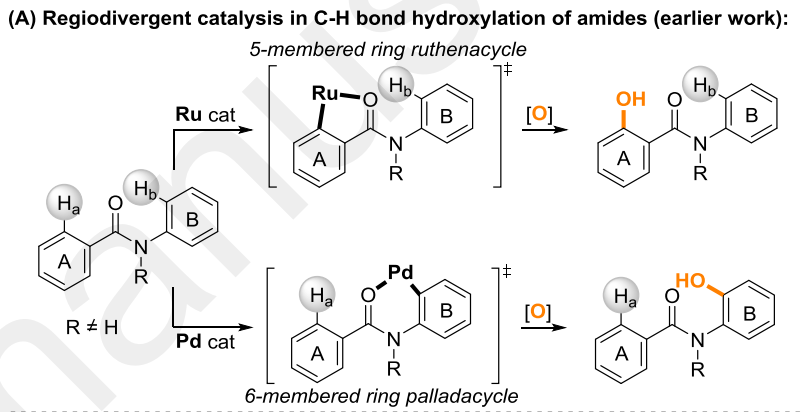

(B) Selective C-H bond hydroxylation of cyclic imides (this approach): distorted 5-membered ring ruthenacycle
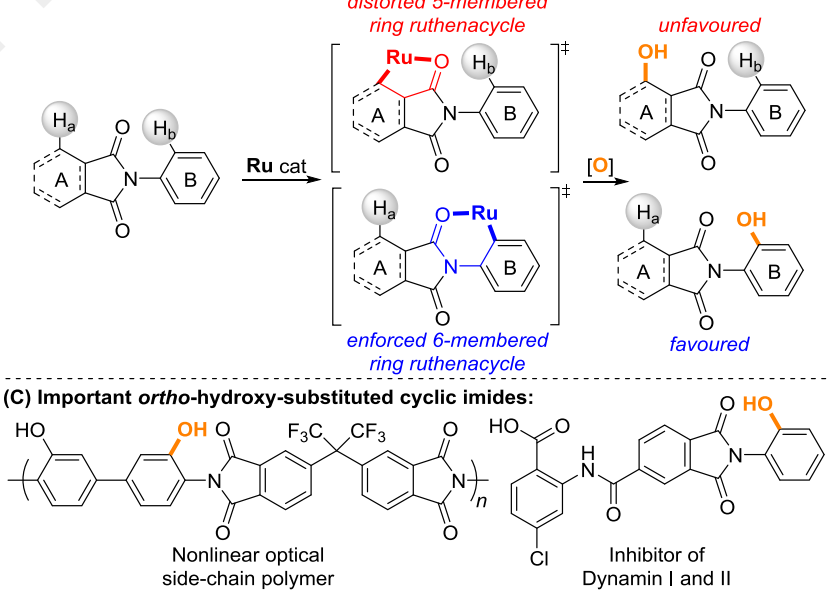

With this in mind, we wondered what the hydroxylation reaction outcome could be by swapping the weak directing group into a more rigid, although synthetically useful, cyclic imides (Scheme 1B). We reasoned that cyclic imides containing two aromatic moieties, such as phthalimide-like molecules, may have difficulties to accommodate a 5-membered ruthenacycle intermediate (activation of $\mathrm{C}-\mathrm{H}_{\mathrm{a}}$ ) due to important ring strain, and consequently, the 6-membered ring (activation of $\mathrm{C}-\mathrm{H}_{\mathrm{b}}$ ) could preferentially form due to the specific pre-disposed orientation of the carbonyl group within the cyclic imide backbone (Scheme 1B). If so, the behavior of the ruthenium catalysts would be the same as the palladium ones for this type of synthetically appealing directing groups in hydroxylation reactions. Herein, we report such strategy, which enables a straightforward access to ortho-hydroxylated cyclic imides together with 
preliminary mechanistic studies to understand the unexpected absence of di-hydroxylated products. $N$-Phenyl-substituted cyclic imides containing hydroxyl groups in the ortho position represent an important class of compounds for polymer sciences ${ }^{9}$ as well as biology and pharmacology (Scheme 1C). ${ }^{10}$ So far, the use of cyclic imides as weak directing groups ${ }^{11}$ in transition metal-catalyzed C$\mathrm{H}$ bond functionalizations has been exclusively limited to selected examples of carbon-carbon bond forming reactions. ${ }^{12}$ Oxygenation reactions via 6-membered ruthenacycle intermediates with 2-pyridyloxy and 2-amino-pyrimidine substituents as strong directing group have been reported respectively.13

\section{RESULTS AND DISCUSSION}

$N$-Phenylphthalimide (1a) was selected as the model substrate for the optimization of the hydroxylation reaction with ruthenium complexes as the pre-catalysts. After screening a number of parameters (Table S1), we found suitable reaction conditions that afforded exclusively the targeted phenol derivative $\mathbf{2 a}$, in which the $\mathrm{C}-\mathrm{H}$ bond oxidation occurred in the hydrogen atom $\mathrm{Hb}_{\mathrm{b}}$ (ortho position with respect to the nitrogen atom), with no evidence of the $\mathrm{C}-\mathrm{H}$ bond functionalization taking place at the other possible hydrogen atom $\mathrm{H}_{\mathrm{a}}$ (Table 1). The reaction conditions consist of 1 or $2.5 \mathrm{~mol} \%$ of $\left[\mathrm{RuCl}_{2}(p \text {-cymene })\right]_{2},\left(\mathrm{NH}_{4}\right)_{2} \mathrm{~S}_{2} \mathrm{O}_{8}$ as oxidant in a mixture of TFA/TFAA $($ TFA $=$ trifluoroacetic acid, TFAA $=$ trifluoroacetic anhydride) as solvents at $80^{\circ} \mathrm{C}$ during $15 \mathrm{~h}$ (Table 1, entries 1-2). In this way, $\mathbf{2 a}$ was obtained in $82 \%$ isolated yield and the same results were obtained when conducting the catalysis at large scale (starting with $2 \mathrm{mmol}$ of $\mathbf{1 a}$ ). In the presence of $\left[\mathrm{RuCl}_{3} \cdot \mathrm{xH}_{2} \mathrm{O}\right]$, the reaction afforded 2a in only 39\% yield (Table 1, entry 3 ); whereas the reaction using $\left[\mathrm{Ru}\left(\mathrm{O}_{2} \mathrm{CMes}\right)_{2}(p\right.$-cymene $\left.)\right]\left(\mathrm{O}_{2} \mathrm{CMes}=2\right.$-mesitylenecarboxylate anion) gave the same yield as using $\left[\mathrm{RuCl}_{2}(p-c y-\right.$ mene) $]_{2}$ (Table 1, entry 4). 1a was unreactive in the absence of any ruthenium complex (Table 1, entry 5); and other oxidants and solvents did not lead to better results (Table S1). Shortening the time $(8 \mathrm{~h})$ and decreasing the temperature $\left(60^{\circ} \mathrm{C}\right)$ of the reaction, respectively, led to lower yields of $\mathbf{2 a}$ (Table 1, entries 6-7). On the other hand, raising the temperature of the reaction to $100{ }^{\circ} \mathrm{C}$ provided similar yields of $\mathbf{2 a}$ as when conducted at $80^{\circ} \mathrm{C}$ (Table 1, entry 8). In the absence of TFAA, almost no reaction took place (Table 1, entry 9) which indicated the key role of TFAA as a source of trifluoroacetate anions during the catalytic cycle..$^{5-8}$ Interestingly, the use of over-stoichiometric amounts of the oxidant did not afford any di-hydroxylation product during the catalysis (Table 1, entry 10). The mono-selectivity observed in this reaction contrasts with other types of carbonyl-containing substrates that do afford di-hydroxylated products as side-products at some extent. ${ }^{7}$ Using $\left[\mathrm{Pd}(\mathrm{OAc})_{2}\right]$ as the pre-catalyst provided 2a in $84 \%$ yield (Table 1, entry 11), showing that the selectivity is exclusively controlled by the structure and coordinating properties of the directing group as it was anticipated (Scheme 1B). From a sustainable and economic point of view, however, ruthenium seems more appealing than palladium for this type of transformations.

The scope of the reaction was evaluated with different $N$-substituted cyclic imides (Scheme 2). para-Substituted N-phenylphthalimides containing different functional groups such as methyl, diethylamino, fluoro, chloro, bromo, iodo and ester followed the protocol leading to the corresponding phenols $\mathbf{2 a - 2 h}$ in $80-90 \%$ isolated yields, respectively. The molecular structure of $\mathbf{2 b}$ and $\mathbf{2 d}$ was further established by single crystal X-ray diffraction studies. The very electron withdrawing group nitro provided the corresponding phenol $2 \mathbf{i}$ in a moderate yield of $53 \%$. N-Phenylphthalimides substituted with bromo and iodo groups in the meta position selectively afforded the phenols $\mathbf{2} \mathbf{j}$ and $\mathbf{2 k}$ in $75 \%$ and $87 \%$ yields, respectively. The reaction was sensitive to the ortho substitution pattern of the $N$-phenyl side of the substrates. For instance, $N$-phenylphthalimide containing the small fluoro substituent at the ortho position afforded the corresponding phenol $2 \mathbf{l}$ in

\section{Table 1. Optimization of the Reaction Conditions ${ }^{a}$}

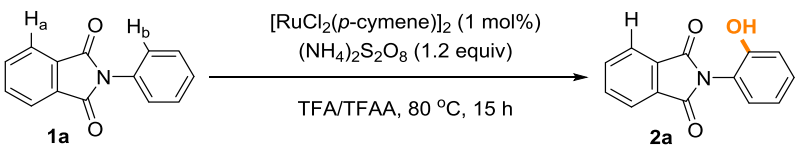

$\begin{array}{llc}\text { entry } & \text { deviation from standard conditions } & \mathbf{2 a}(\%)^{\boldsymbol{b}} \\ 1 & \text { none } & 85(82)^{\boldsymbol{c}} \\ 2 & 2.5 \mathrm{~mol} \% \text { of }\left[\mathrm{RuCl}_{2}(p \text {-cymene })\right]_{2} & 83 \\ 3 & 2.5 \mathrm{~mol} \%\left[\mathrm{RuCl}_{3} \cdot \mathrm{xH}_{2} \mathrm{O}\right] & 39 \\ 4 & 2.5 \mathrm{~mol} \%\left[\mathrm{Ru}\left(\mathrm{O}_{2} \mathrm{CMes}_{2}(p \text {-cymene })\right]\right. & 86 \\ 5 & \text { no }\left[\mathrm{RuCl}_{2}(p \text {-cymene })\right]_{2} & 0 \\ 6 & 8 \mathrm{~h} \mathrm{instead} \mathrm{of} 15 \mathrm{~h} & 79 \\ 7 & 60{ }^{\circ} \mathrm{C} \text { instead of } 80{ }^{\circ} \mathrm{C} & 50 \\ 8 & 100{ }^{\circ} \mathrm{C} \text { instead of } 80{ }^{\circ} \mathrm{C} & 85 \\ 9 & \text { no TFAA } & <5 \\ 10 & 2-10 \text { equiv of }\left(\mathrm{NH}_{4}\right)_{2} \mathrm{~S}_{2} \mathrm{O}_{8} & 85 \\ 11 & {\left[\mathrm{Pd}(\mathrm{OAc})_{2}\right] \text { instead of }\left[\mathrm{RuCl}_{2}(p \text {-cymene })\right]_{2}} & 80^{d}\end{array}$

${ }^{a} \mathbf{1 a}(0.1 \mathrm{mmol}),\left(\mathrm{NH}_{4}\right)_{2} \mathrm{~S}_{2} \mathrm{O}_{8}(0.12 \mathrm{mmol}),\left[\mathrm{RuCl}_{2} \text { (p-cymene) }\right]_{2}(1 \mathrm{~mol} \%)$ in TFA/TFAA $(0.5 \mathrm{~mL}, v / v 3: 1)$ at $80^{\circ} \mathrm{C}$ for $15 \mathrm{~h}$ under air. ${ }^{b}$ Yield calculated by ${ }^{1} \mathrm{H}$ NMR spectroscopy analysis using dibromomethane as internal standard. ${ }^{c}$ Isolated yield. ${ }^{d} 2 \mathrm{~mol} \%$.

\section{Scheme 2. Substrate Scope ${ }^{a, b}$}

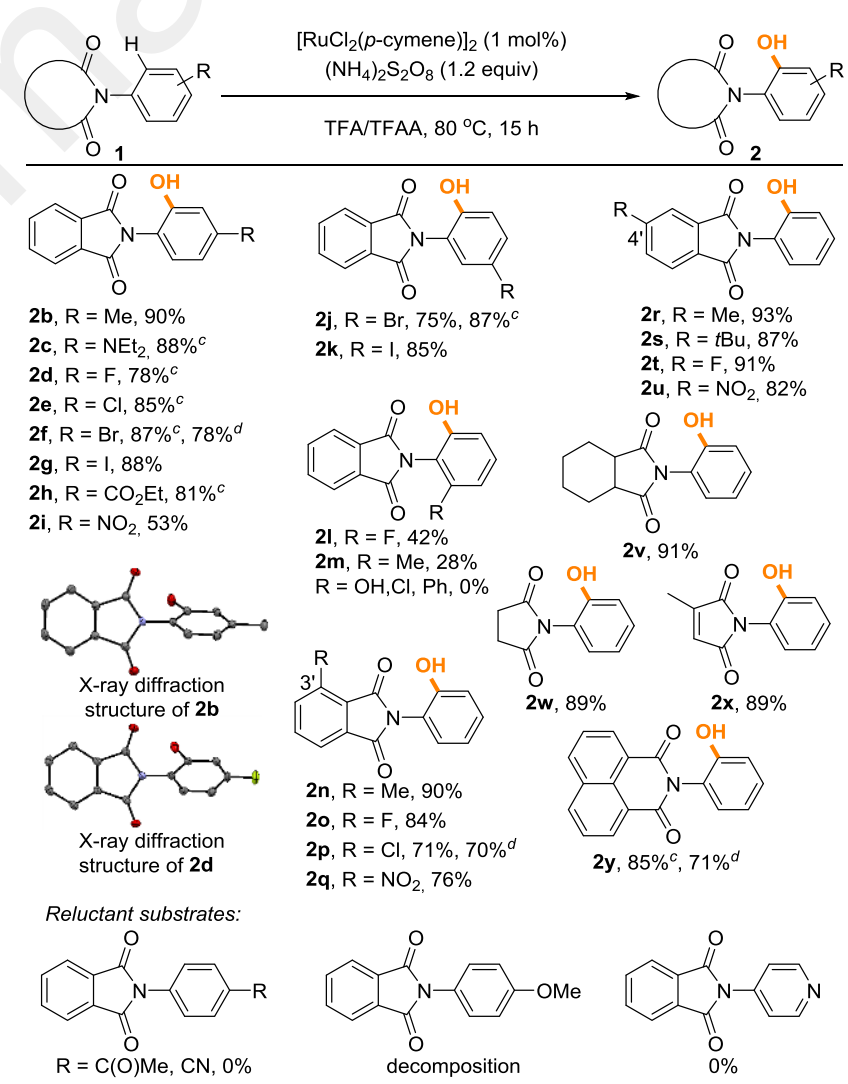

${ }^{a}$ Reaction conditions: $1(0.3 \mathrm{mmol}),\left(\mathrm{NH}_{4}\right)_{2} \mathrm{~S}_{2} \mathrm{O}_{8}(0.36 \mathrm{mmol}),\left[\mathrm{RuCl}_{2}(p\right.$-cymene $)]_{2}(1 \mathrm{~mol} \%)$ in TFA/TFAA $(1 \mathrm{~mL}, v / v 3: 1)$ at $80^{\circ} \mathrm{C}$ for $15 \mathrm{~h}$ under air. ${ }^{b}$ Isolated yields. ${ }^{c} 2.5 \mathrm{~mol} \%$ of $\left[\mathrm{RuCl}_{2}(p \text {-cymene })\right]_{2} \cdot{ }^{d}\left[\mathrm{Pd}(\mathrm{OAc})_{2}\right](2 \mathrm{~mol} \%)$ instead of $\left[\mathrm{RuCl}_{2}(p \text {-cymene })\right]_{2}(1 \mathrm{~mol} \%)$. 
$42 \%$ yield, whereas a substrate with the methyl group afforded the phenol $\mathbf{2 m}$ in only $28 \%$ yield. No reaction was observed for substrates containing the hydroxyl, chloro and phenyl substituents at the ortho position. The reaction of ortho-substituted phthalimides seem to be affected not only by the bulkiness of the substituents but also by the electrostatic repulsion between the substituents and the imide carbonyl group. In addition, there was no impact for the reactivity of $\mathrm{N}$-phenylphthalimides substituted at 3' or 4' position with different functional groups such as alkyl $(\mathrm{Me}, t \mathrm{Bu})$, halide $(\mathrm{F}$,

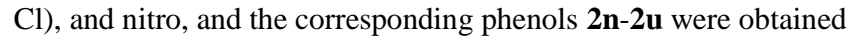
in 71-93\% yields. Interestingly, other cyclic imides behaved as excellent directing groups as the phthalimides. A cyclohexane-containing cyclic imide afforded the corresponding phenol $\mathbf{2 v}$ in $91 \%$ yield and, $\mathrm{N}$-phenyl-substituted succinimide and 2-methylmaleimide gave rise to the corresponding phenols $\mathbf{2} \mathbf{w}$ and $\mathbf{2 x}$ in $89 \%$ yield in both cases. The catalysis was also applicable ( $85 \%$ yield) to $N$-phenylnaphthalimide (2y), which is an appealing motif for material sciences. ${ }^{14}$ Similar reactivity was observed using $2 \mathrm{~mol} \%$ of $\left[\mathrm{Pd}(\mathrm{OAc})_{2}\right]$ instead of $\left[\mathrm{RuCl}_{2}(p \text {-cymene })\right]_{2}$ as the pre-catalyst as it was exemplified in the synthesis of phenols $2 \mathbf{a}, \mathbf{2 f}, \mathbf{2 p}$, and $\mathbf{2 y}$ in $70-80 \%$ isolated yields (Scheme 2). In all cases (2a-2y), no di-hydroxylated products were observed. Unfortunately, no reactivity was found for $N$-(4-acetylphenyl)phthalimide, $N$-(4-cyanophenyl)phthalimide and $N$-(4-pyridyl)phthalimide (Scheme 2 ) neither for $\mathrm{C}\left(\mathrm{sp}^{3}\right)-\mathrm{H}$ bond hydroxylation of $N$-alkylphthalimides. $N-(4-$ methoxyphenyl)phthalimide decomposed under the studied reaction conditions leading to an unidentified mixture of species (Scheme 2).

To gain further insights into the reaction mechanism, we performed the catalysis with substrates lacking one and two carbonyl groups, respectively (Eq. 1-2). Under the standard reaction conditions, the selective mono-hydroxylation reaction took place with a cyclic amide ( $81 \%$ yield of $\mathbf{3}$, Eq. 1) with traces of the di-hydroxylated product 4 detected by TLC and GC-MS analysis. However, increasing the number of equivalents of oxidant to three afforded exclusively the di-hydroxylated product 4 in $82 \%$ yield (Eq. 1) whereas the excess of oxidant has no impact when a cyclic imide was used (Table 1, entry 10). On the other hand, no reaction occurred with a cyclic amine (Eq. 2), which indicates the coordinating role of the carbonyl group throughout the catalytic cycle. Consequently, the selectivity observed for di- $v s$ mono-hydroxylation in the resulting products is likely controlled by an increase in the steric bulk when going from cyclic amides to cyclic imides as the weak directing groups.

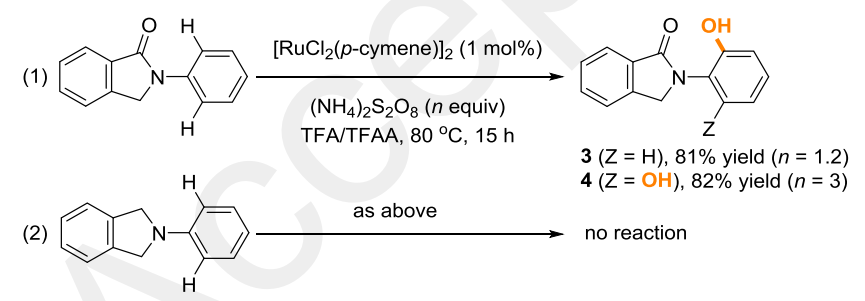

The above described findings together with previous contributions $^{5-8}$ enabled us to propose a catalytic cycle (Figure 1a). Initially, one of the carbonyl groups of the imide substrate coordinated to a chloride-free ruthenium species leading to $\mathbf{A}$ that followed $\mathrm{C}-\mathrm{H}$ bond activation at the ortho position to form the 6-membered ruthenacycle intermediate $\mathbf{B}$. After oxidation and reductive elimination, the trifluoroacetate product $\mathbf{D}$ was formed followed by hydrolysis releasing the final product $\mathbf{2}$. The nature of $\mathbf{D}$ was evidenced by GC-MS analysis of the crude reaction mixture using 1a as substrate, which showed a peak at $m / z=335$ corresponding to the trifluoroacetate-containing $N$-phenylphthalimide (Figure $\mathrm{S} 1$ in the
Supporting Information). After hydrolysis, this peak disappeared and the peak corresponding to the phenol $\mathbf{2 a}(\mathrm{m} / \mathrm{z}=239)$ was observed (Figure S2 in the Supporting Information). The origin of the selectivity for the exclusive mono-hydroxylation and the fact that the catalysis was sensitive to the ortho substitution pattern was addressed as well (Figure 1b). The rotational barrier around the $\mathrm{N}$ phenyl axis of non-substituted $N$-phenylphthalimide was estimated to be $c a .10 \mathrm{~kJ} / \mathrm{mol}$ (see Supporting Information), which suggests a fast interconversion between the staggered and the eclipsed conformations. This translates into an accessible pathway (blue arrows, Figure 1b) for the catalytically productive intermediate $\mathbf{A}_{\mathbf{E}}$ (where the $\mathrm{C}-\mathrm{H}$ bond is in close proximity of the ruthenium center) and ruthenacycle $\mathbf{B}_{\mathbf{E}}$ (co-planar 6-membered ring). On the other hand, a substitution pattern in the ortho position favors the staggered conformation at a higher extent with a rotational barrier around the $\mathrm{N}$ phenyl axis estimated to be, at least, higher than $20 \mathrm{~kJ} / \mathrm{mol}$ for $\mathrm{R}=$ $\mathrm{OH}$ or $\mathrm{OC}(\mathrm{O}) \mathrm{CF}_{3}$ (see Supporting Information). Consequently, the catalytically unproductive intermediates As (where the C-H bond is far away from the ruthenium center) and Bs (distorted 6-membered ruthenacycle) dominate in this catalytically unproductive case (red arrows, Figure 1b).

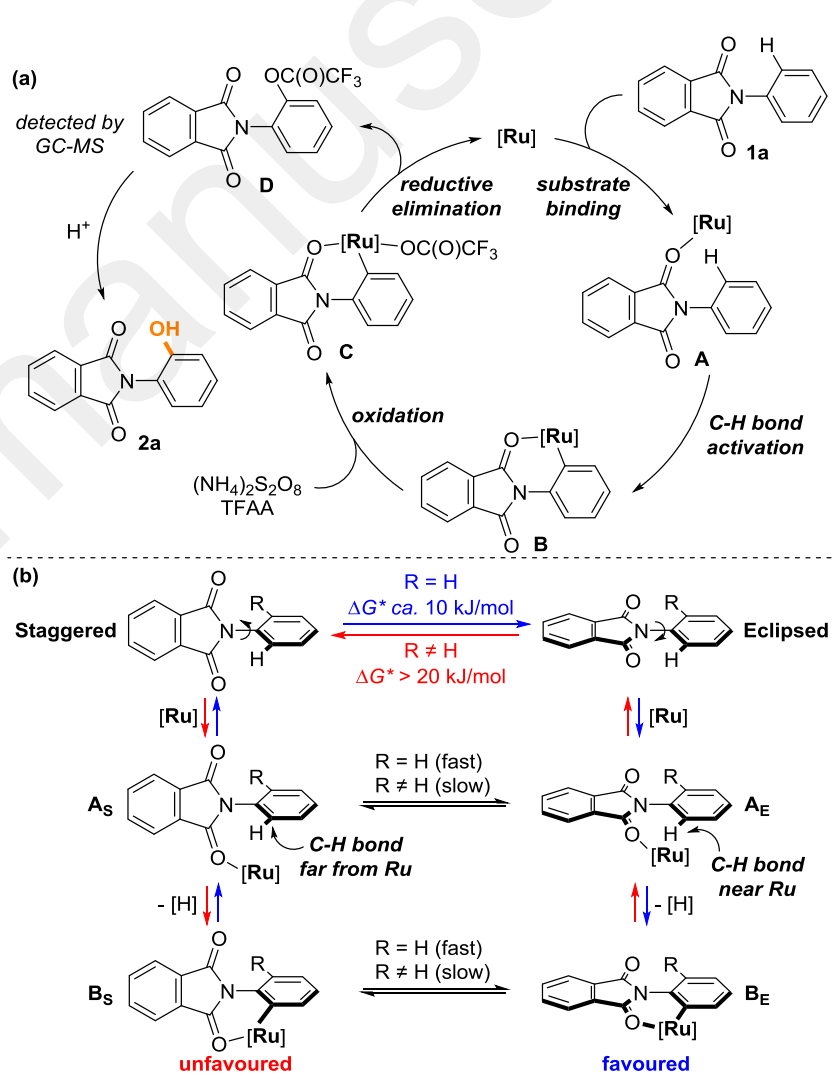

Figure 1. (a) Proposed catalytic cycle. (b) Stereochemical model explaining the selectivity for mono-hydroxylation.

Finally, a brief applicability of the transformation was demonstrated (Scheme 3). The ortho-hydroxylated phthalimides were alkylated leading to the methoxy-containing product $\mathbf{5}$ in almost quantitative yield. They could be transformed into a benzoxazole upon thermal treatment ${ }^{15}$ and into amide 6 via ruthenium catalsyis. ${ }^{\mathbf{1 6}}$ Deprotection with hydrazine led to the corresponding 2 -aminoanisole (7), highlighting the great potential of the phthalimide ring as a traceless directing group for the transition metal-catalyzed $\mathrm{C}-\mathrm{H}$ bond functionalizations. 


\section{Scheme 3. Postfunctionalization Reactions ${ }^{a}$}

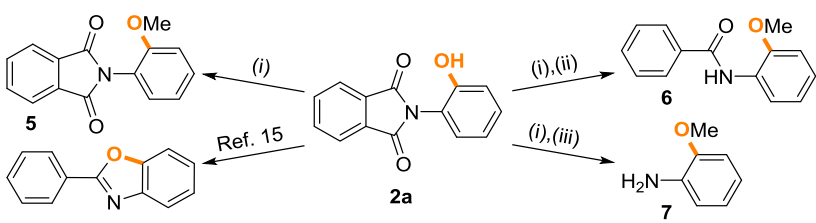

${ }^{a}$ Reaction conditions: (i) $\mathrm{MeI}$ (1.5 equiv), $\mathrm{K}_{2} \mathrm{CO}_{3}$ (1.5 equiv), $\mathrm{DMF}(0.2 \mathrm{M})$, $20{ }^{\circ} \mathrm{C}, 2 \mathrm{~h}, 96 \%$ yield; (ii) $\mathrm{H}_{2} \mathrm{O}$ (1.5 equiv), $\mathrm{K}_{2} \mathrm{CO}_{3}$ (3 equiv), $\left[\mathrm{RuCl}_{2}(p\right.$ cymene $)]_{2}(5 \mathrm{~mol} \%)$, NMP $(0.2 \mathrm{M}), 150{ }^{\circ} \mathrm{C}, 48 \mathrm{~h}, 78 \%$ yield; (iii) $\mathrm{N}_{2} \mathrm{H}_{4} \cdot \mathrm{H}_{2} \mathrm{O}$ (7.75 equiv), EtOH (0.2 M), reflux, 2 h, $83 \%$ yield.

\section{- CONCLUSION}

In summary, we have developed a selective $\mathrm{C}\left(\mathrm{sp}^{2}\right)-\mathrm{H}$ bond hydroxylation reaction of a large variety of functionalized phthalimides, succinimides, maleimides, naphthalimides, and cyclic amides. The reactions proceeded under relatively mild conditions with as low as $1 \mathrm{~mol} \%$ of readily-available ruthenium or palladium catalysts affording the corresponding mono-hydroxylated products at the ortho position with respect to the nitrogen atom of the directing group. As such, cyclic imides can now be further exploited as easily-removable, weak directing groups for other types of $\mathrm{C}-\mathrm{H}$ bond functionalizations leading to useful shortcuts in the synthesis of fine chemicals.

\section{- EXPERIMENTAL SECTION}

General information. All reagents were obtained from commercial sources and used as supplied. All reactions were carried out in flame-dried glassware under argon atmosphere unless otherwise noted. Catalytic experiments were performed in Schlenk-type flasks under argon atmosphere unless otherwise noted. Organic solutions were concentrated under reduced pressure using a rotary evaporator. Thin-layer chromatography (TLC) were carried out on $0.25 \mathrm{~mm}$ Merck silica gel (60-F254). Flash column chromatography was performed using silica gel Silica $60 \mathrm{M}, 0.04-0.063 \mathrm{~mm}$. Technical grade petroleum ether (40-60), $n$-heptane and ethyl acetate were used for column chromatography. $\mathrm{CDCl}_{3}$ was stored under nitrogen over molecular sieves. NMR spectra were recorded on an AVANCE III 400 spectrometer. ${ }^{1} \mathrm{H}$ NMR spectra were referenced to residual protiated solvent $\left(\delta=7.26 \mathrm{ppm}\right.$ for $\mathrm{CDCl}_{3}, \delta=$ $2.50 \mathrm{ppm}$ for DMSO- $d_{6}$ and $\delta=2.05 \mathrm{ppm}$ for acetone- $d_{6}$ ) and ${ }^{13} \mathrm{C}$ chemical shifts are reported relative to deuterated solvents $(\delta=77.0$ ppm for $\mathrm{CDCl}_{3}, \delta=39.5 \mathrm{ppm}$ for DMSO- $d_{6}$ and $\delta=29.8 \mathrm{ppm}$ for acetone- $d_{6}$ ) [Note: acetone- $d_{6}$ contains traces of water at $c a .3$ $\mathrm{ppm}$. The peak patterns are indicated as follows: s, singlet; d, doublet; $\mathrm{t}$, triplet; q, quartet; $\mathrm{m}$, multiplet, and br. for broad. GC-MS analyses were performed with a GCMS-QP2010S (Shimadzu) instrument with a GC-2010 equipped with a $30 \mathrm{~m}$ capillary column (Supelco, SLBTM-5ms, fused silica capillary column, $30 \mathrm{~m} \times 0.25$ $\mathrm{mm} \times 0.25 \mathrm{~mm}$ film thickness), which was used with helium as the vector gas. The following GC conditions were used: initial temperature $80^{\circ} \mathrm{C}$ for 2 minutes, then rate $20^{\circ} \mathrm{C} /$ min until $280{ }^{\circ} \mathrm{C}$ and 280 ${ }^{\circ} \mathrm{C}$ for 28 minutes. HRMS were recorded on a Waters Q-Tof 2 mass spectrometer at the corresponding facilities of the CRMPO, Centre Régional de Mesures Physiques de l'Ouest, Université de Rennes 1.

Synthesis and characterization of substrates (1). Method A: Phthalic anhydride (5 mmol, $0.74 \mathrm{~g}, 1$ eq.) and the corresponding aniline (5 mmol, 1 eq.) were refluxed in acetic acid $(30 \mathrm{~mL})$ for 25 hours. Once at room temperature, water was added and the solid recovered by filtration. After drying under vacuum the desired phthalimide was obtained. Method B: A mixture of 2-formylbenzoic acid (5.0 mmol, 1 eq.), amine (6.0 mmol, 1.2 eq.), DABCO (10.0 mmol, 2 eq.), HCOOH (1.25 mL), Pd(OAc) 2 (0.25 mmol, 5 $\mathrm{mol} \%)$ in 1,4-dioxane $(5 \mathrm{~mL})$ was heated to $80{ }^{\circ} \mathrm{C}$ for $3 \mathrm{~h}$. After completion of the reaction, the mixture was cooled to room temperature, and diluted with DCM $(50 \mathrm{~mL})$. The solid was removed by filter, and the filtrate was washed with water $(50 \mathrm{~mL})$ and brine $(50 \mathrm{~mL})$. The organic layer was dried over $\mathrm{Na}_{2} \mathrm{SO}_{4}$, filtered, and concentrated under reduced pressure. The residue was purified by column chromatography on silica gel (petroleum ether/acetone $=$ $5 / 1, v / v)$ to afford the desired product. Method C: $1,2-\mathrm{Bis}$ (bromomethyl)benzene (5.0 mmol, 1 eq.), DIPEA (12.5 mmol, 2.5 eq.), and aniline ( $7.50 \mathrm{mmol}, 1.5 \mathrm{eq}$.) dissolved in toluene $(25 \mathrm{~mL})$ were added to a tube sealing before vigorously stirring at $110^{\circ} \mathrm{C}$ under a $\mathrm{Ar}$ atmosphere. The resulting mixture was cooled to room temperature and extracted with ethyl acetate $(3 \times 10 \mathrm{~mL})$. The combined organic phase was washed with brine, dried over anhydrous $\mathrm{Na}_{2} \mathrm{SO}_{4}$ and concentrated in vacuo. The crude product was purified by column chromatography (petroleum ether) to obtain the desired product as a light yellow solid. Method D: Hexahydrophthalic anhydride (10 mmol, $1.54 \mathrm{~g}, 1$ eq.), aniline (10 mmol, 1 eq.) and THF $(15 \mathrm{~mL})$ were added to a $100 \mathrm{~mL}$ round bottom flask. The solution was stirred for $30 \mathrm{~min}$ at $40^{\circ} \mathrm{C}$. Removal of the solvent using a rotary evaporator gave the corresponding carboxylic acid-amide as white solid. The white solid was then heated at $190{ }^{\circ} \mathrm{C}$ under Ar for $4 \mathrm{~h}$. The desired phthalimide was purified by silica gel column chromatography with a mixture of petroleum ether and ethyl acetate as eluent. Method E: A solution of succinic acid (10 mmol, 1 eq.) and aniline (10 mmol, 1 eq.) were dissolved in water $(5.0 \mathrm{~mL})$ in a flask and stirred and maintained at boiling for $2 \mathrm{~h}$. The reaction progress was monitored by TLC (1:1 $n$-hexane/acetone). The reaction mixture was cooled to room temperature and the product was filtered, washed with water and recrystallized from methanol.

$\mathrm{N}$-Phenylphthalimide (1a). Prepared according to Method A starting from aniline in $80 \%$ isolated yield $(891 \mathrm{mg}) .{ }^{1} \mathrm{H}$ NMR $(400$ $\left.\mathrm{MHz}, \mathrm{CDCl}_{3}\right): \delta=7.96(\mathrm{dd}, J=5.6,3.2 \mathrm{~Hz}, 2 \mathrm{H}), 7.80(\mathrm{dd}, J=5.2$, $3.2 \mathrm{~Hz}, 2 \mathrm{H}), 7.52$ (dd, $J=7.6,7.6 \mathrm{~Hz}, 2 \mathrm{H}), 7.34-7.27$ (m, $3 \mathrm{H}) \mathrm{ppm}$. The spectral data match those previously reported. ${ }^{17}$

$\mathrm{N}$-( $p$-Tolyl)phthalimide ( $\mathbf{1 b})$. Prepared according to Method A starting from $p$-toluidine in $72 \%$ isolated yield $(1.2 \mathrm{~g}) .{ }^{1} \mathrm{H}$ NMR (400 MHz, $\left.\mathrm{CDCl}_{3}\right): \delta=7.94(\mathrm{dd}, J=5.6,3.2 \mathrm{~Hz}, 2 \mathrm{H}), 7.78(\mathrm{dd}, J$ $=5.6,3.2 \mathrm{~Hz}, 2 \mathrm{H}), 7.31(\mathrm{~s}, 4 \mathrm{H}), 2.41(\mathrm{~s}, 3 \mathrm{H}) \mathrm{ppm}$. The spectral data match those previously reported. ${ }^{\mathbf{1 7}}$

$N-((p$-Diethylamino $)$ phenyl)phthalimide $(1 c)$. Prepared according to Method A starting from $N, N$-diethyl-1,4-phenylenediamine in $90 \%$ isolated yield $(1.58 \mathrm{~g}) .{ }^{1} \mathrm{H} \mathrm{NMR}\left(400 \mathrm{MHz}, \mathrm{CDCl}_{3}\right): \delta=$ $7.93(\mathrm{dd}, J=5.6,3.2 \mathrm{~Hz}, 2 \mathrm{H}), 7.75(\mathrm{dd}, J=5.6,3.2 \mathrm{~Hz}, 2 \mathrm{H}), 7.20$ $(\mathrm{dd}, J=6.8,2.4 \mathrm{~Hz}, 2 \mathrm{H}), 6.73(\mathrm{dd}, J=6.8,2.4 \mathrm{~Hz}, 2 \mathrm{H}), 3.38(\mathrm{q}, J$ $=7.2 \mathrm{~Hz}, 4 \mathrm{H}), 1.19(\mathrm{t}, J=7.2 \mathrm{~Hz}, 6 \mathrm{H}) \mathrm{ppm}$. The spectral data match those previously reported. ${ }^{18}$

$N$-(p-Fluorophenyl)phthalimide (1d). Prepared according to Method A starting from 4-fluoroaniline in $82 \%$ isolated yield (1.59 g). ${ }^{1} \mathrm{H}$ NMR $\left(400 \mathrm{MHz}, \mathrm{CDCl}_{3}\right): \delta=7.86(\mathrm{dd}, J=5.2,3.2 \mathrm{~Hz}, 2 \mathrm{H})$, $7.71(\mathrm{dd}, J=5.2,3.2 \mathrm{~Hz}, 2 \mathrm{H}), 7.34(\mathrm{dd}, J=9.2,4.8 \mathrm{~Hz}, 2 \mathrm{H}), 7.11$ (dd, $J=8.8,8.8 \mathrm{~Hz}, 2 \mathrm{H}) \mathrm{ppm} .{ }^{19} \mathrm{~F}\left\{{ }^{1} \mathrm{H}\right\} \mathrm{NMR}\left(376 \mathrm{MHz}, \mathrm{CDCl}_{3}\right)$ : $\delta=-113.8 \mathrm{ppm}$. The spectral data match those previously reported. ${ }^{19}$

$N$-(p-Chlorophenyl)phthalimide (1e). Prepared according to Method A starting from 4-chloroaniline in 91\% isolated yield (1.17 g). ${ }^{1} \mathrm{H}$ NMR (400 MHz, $\left.\mathrm{CDCl}_{3}\right): \delta=7.96(\mathrm{dd}, J=5.6,3.2 \mathrm{~Hz}, 2 \mathrm{H})$, $7.81(\mathrm{dd}, J=5.6,3.2 \mathrm{~Hz}, 2 \mathrm{H}), 7.48(\mathrm{dd}, J=6.4,2.4 \mathrm{~Hz}, 2 \mathrm{H}), 7.42$ $(\mathrm{dd}, J=6.4,2.4 \mathrm{~Hz}, 2 \mathrm{H}) \mathrm{ppm}$. The spectral data match those previously reported. ${ }^{20}$ 
$N$-(p-Bromophenyl)phthalimide (1f). Prepared according to Method A starting from 4-bromoaniline in $88 \%$ isolated yield (1.61 g). ${ }^{1} \mathrm{H} \mathrm{NMR}\left(400 \mathrm{MHz}, \mathrm{CDCl}_{3}\right): \delta=7.96(\mathrm{dd}, J=5.2,2.8 \mathrm{~Hz}, 2 \mathrm{H})$, $7.80(\mathrm{dd}, J=5.2,2.8 \mathrm{~Hz}, 2 \mathrm{H}), 7.63(\mathrm{~d}, J=8.4 \mathrm{~Hz}, 2 \mathrm{H}), 7.36(\mathrm{~d}, J$ $=8.8 \mathrm{~Hz}, 2 \mathrm{H}) \mathrm{ppm}$. The spectral data match those previously reported. ${ }^{21}$

$N$-(p-Iodophenyl)phthalimide (1g). Prepared according to Method A starting from 4-iodoaniline in $80 \%$ isolated yield (1.39 g). ${ }^{1} \mathrm{H}$ NMR $\left(400 \mathrm{MHz}, \mathrm{CDCl}_{3}\right): \delta=7.96-7.94(\mathrm{~m}, 2 \mathrm{H}), 7.84-7.79$ $(\mathrm{m}, 4 \mathrm{H}), 7.23(\mathrm{~d}, J=8.4 \mathrm{~Hz}, 2 \mathrm{H}) \mathrm{ppm}$. The spectral data match those previously reported. ${ }^{22}$

$N$-(p-Ethoxycarbonylphenyl)phthalimide (1h). Prepared according to Method A starting from benzocaine in 50\% isolated yield (801 mg). ${ }^{1} \mathrm{H} \mathrm{NMR}\left(400 \mathrm{MHz}, \mathrm{CDCl}_{3}\right): \delta=8.19$ (dd, $J=6.8$, $1.6 \mathrm{~Hz}, 2 \mathrm{H}), 7.97(\mathrm{dd}, J=5.2,2.8 \mathrm{~Hz}, 2 \mathrm{H}), 7.82(\mathrm{dd}, J=5.2,2.8$ $\mathrm{Hz}, 2 \mathrm{H}), 7.59(\mathrm{dd}, J=6.8,1.6 \mathrm{~Hz}, 2 \mathrm{H}), 4.41(\mathrm{q}, J=7.2 \mathrm{~Hz}, 2 \mathrm{H})$, $1.41(\mathrm{t}, J=7.2 \mathrm{~Hz}, 3 \mathrm{H}) \mathrm{ppm}$. The spectral data match those previously reported. ${ }^{23}$

$N$-(p-Nitrophenyl)phthalimide (1i). Prepared according to Method A starting from 4-nitroaniline in 63\% isolated yield (758 mg). ${ }^{1} \mathrm{H}$ NMR $\left(400 \mathrm{MHz}, \mathrm{CDCl}_{3}\right): \delta=8.38(\mathrm{dd}, J=7.2,2.0 \mathrm{~Hz}$, $2 \mathrm{H}), 8.00(\mathrm{dd}, J=5.2,2.8 \mathrm{~Hz}, 2 \mathrm{H}), 7.85(\mathrm{dd}, J=5.6,3.2 \mathrm{~Hz}, 2 \mathrm{H})$, $7.78(\mathrm{dd}, J=7.2,2.0 \mathrm{~Hz}, 2 \mathrm{H}) \mathrm{ppm}$. The spectral data match those previously reported. ${ }^{17}$

$N$-(m-Bromophenyl)phthalimide $(\mathbf{1 j})$. Prepared according to Method A starting from 3-bromoaniline in 70\% isolated yield (1.69 g). ${ }^{1} \mathrm{H} \mathrm{NMR}\left(400 \mathrm{MHz}, \mathrm{CDCl}_{3}\right): \delta=7.96(\mathrm{dd}, J=5.6,3.2 \mathrm{~Hz}, 2 \mathrm{H})$, $7.80(\mathrm{dd}, J=5.6,3.2 \mathrm{~Hz}, 2 \mathrm{H}), 7.65(\mathrm{dd}, J=2.0,2.0 \mathrm{~Hz}, 1 \mathrm{H}), 7.55-$ $7.52(\mathrm{~m}, 1 \mathrm{H}), 7.44-7.41(\mathrm{~m}, 1 \mathrm{H}), 7.37(\mathrm{dd}, J=8.0,8.0 \mathrm{~Hz}, 1 \mathrm{H})$ ppm. The spectral data match those previously reported. ${ }^{24}$

$N$-(m-Iodophenyl)phthalimide $(\mathbf{1 k})$. Prepared according to Method A starting from 3-iodoaniline in $88 \%$ isolated yield (1.50 g). ${ }^{1} \mathrm{H} \mathrm{NMR}\left(400 \mathrm{MHz}, \mathrm{CDCl}_{3}\right): \delta=7.97(\mathrm{dd}, J=5.6,3.2 \mathrm{~Hz}, 2 \mathrm{H})$, 7.83-7.80 (m, 3H), 7.74 (ddd, $J=8.0,2.4,2.4 \mathrm{~Hz}, 1 \mathrm{H}), 7.45$ (dd, $J$ $=8.0,2.4 \mathrm{~Hz}, 1 \mathrm{H}), 7.24(\mathrm{dd}, J=8.0,8.0 \mathrm{~Hz}, 1 \mathrm{H}) \mathrm{ppm}$. The spectral data match those previously reported. ${ }^{21}$

$\mathrm{N}$-(o-Fluorophenyl)phthalimide (1l). Prepared according to Method A starting from 2-fluoroaniline in 91\% isolated yield (1.53 g). ${ }^{1} \mathrm{H} \mathrm{NMR}\left(400 \mathrm{MHz}, \mathrm{CDCl}_{3}\right): \delta=7.97(\mathrm{dd}, J=5.6,3.2 \mathrm{~Hz}, 2 \mathrm{H})$, $7.81(\mathrm{dd}, J=5.2,3.2 \mathrm{~Hz}, 2 \mathrm{H}), 7.48-7.43(\mathrm{~m}, 1 \mathrm{H}), 7.39-7.35(\mathrm{~m}$, 1H), 7.31-7.25 (m, 2H) ppm. ${ }^{19} \mathrm{~F}\left\{{ }^{1} \mathrm{H}\right\}$ NMR (376 MHz, $\left.\mathrm{CDCl}_{3}\right): \delta$ $=-118.7 \mathrm{ppm}$. The spectral data match those previously reported. ${ }^{25}$

$\mathrm{N}$-o-Tolylphthalimide $(\mathbf{1 m})$. Prepared according to Method A starting from $o$-toluidine in $43 \%$ isolated yield $(1.2 \mathrm{~g}) .{ }^{1} \mathrm{H}$ NMR $\left(400 \mathrm{MHz}, \mathrm{CDCl}_{3}\right): \delta=7.96(\mathrm{dd}, J=5.6,3.2 \mathrm{~Hz}, 2 \mathrm{H}), 7.80(\mathrm{dd}, J$ $=5.6,2.8 \mathrm{~Hz}, 2 \mathrm{H}), 7.39-7.31(\mathrm{~m}, 3 \mathrm{H}), 7.21(\mathrm{~d}, J=7.6 \mathrm{~Hz}, 1 \mathrm{H})$, $2.21(\mathrm{~s}, 3 \mathrm{H}) \mathrm{ppm}$. The spectral data match those previously reported. ${ }^{26}$

N-Phenyl-3-methylphthalimide (1n). Prepared according to Method A starting from 3-methylphthalic anhydride in 95\% isolated yield $(1.13 \mathrm{~g}) .{ }^{1} \mathrm{H}$ NMR $\left(400 \mathrm{MHz}, \mathrm{CDCl}_{3}\right): \delta=7.78(\mathrm{~d}, J=$ $7.2 \mathrm{~Hz}, 1 \mathrm{H}), 7.63(\mathrm{dd}, J=7.6 \mathrm{~Hz}, 1 \mathrm{H}), 7.54-7.49(\mathrm{~m}, 3 \mathrm{H}), 7.45-$ $7.38(\mathrm{~m}, 3 \mathrm{H}), 2.75(\mathrm{~s}, 3 \mathrm{H}) \mathrm{ppm}$. The spectral data match those previously reported. ${ }^{27}$

N-Phenyl-3-fluorophthalimide (1o). Prepared according to Method A starting from 3-fluorophthalic anhydride in $91 \%$ isolated yield (1.09 g). ${ }^{1} \mathrm{H}$ NMR (400 MHz, $\left.\mathrm{CDCl}_{3}\right): \delta=7.82-7.77(\mathrm{~m}, 2 \mathrm{H})$, 7.53-7.49 (m, 2H), 7.47-7.40 (m, 4H) ppm. ${ }^{19} \mathrm{~F}\left\{{ }^{1} \mathrm{H}\right\}$ NMR $(376$ $\left.\mathrm{MHz}, \mathrm{CDCl}_{3}\right): \delta=-112.3 \mathrm{ppm}$. The spectral data match those previously reported. ${ }^{28}$

$N$-Phenyl-3-chlorophthalimide (1p). Prepared according to Method A starting from 3-chlorophthalic anhydride in 94\% isolated yield $(1.2 \mathrm{~g}) .{ }^{1} \mathrm{H}$ NMR (400 $\left.\mathrm{MHz}, \mathrm{CDCl}_{3}\right): \delta=7.87(\mathrm{dd}, J=4.8$, $3.6 \mathrm{~Hz}, 1 \mathrm{H}), 7.71-7.70(\mathrm{~m}, 2 \mathrm{H}), 7.53-7.48(\mathrm{~m}, 2 \mathrm{H}), 7.45-7.41(\mathrm{~m}$, $3 \mathrm{H}) \mathrm{ppm}$. The spectral data match those previously reported. ${ }^{29}$
N-Phenyl-3-nitrophthalimide (1q). Prepared according to Method A starting from 3-nitrophthalic anhydride in 78\% isolated yield (972 mg). ${ }^{1} \mathrm{H}$ NMR (400 MHz, $\mathrm{CDCl}_{3}$ ): $\delta=8.21$ (dd, $J=7.6$, $0.8 \mathrm{~Hz}, 1 \mathrm{H}), 8.15(\mathrm{dd}, J=8.0,0.8 \mathrm{~Hz}, 1 \mathrm{H}), 7.98(\mathrm{dd}, J=8.0,8.0$ $\mathrm{Hz}, 1 \mathrm{H}), 7.54-7.50(\mathrm{~m}, 2 \mathrm{H}), 7.46-7.42(\mathrm{~m}, 3 \mathrm{H}) \mathrm{ppm}$. The spectral data match those previously reported..$^{30}$

N-Phenyl-4-methylphthalimide (1r). Prepared according to Method A starting from 4-methylphthalic anhydride in $97 \%$ isolated yield (1.15 g). ${ }^{1} \mathrm{H} \mathrm{NMR}\left(400 \mathrm{MHz}, \mathrm{CDCl}_{3}\right): \delta=7.83(\mathrm{~d}, J=$ $7.6 \mathrm{~Hz}, 1 \mathrm{H}), 7.75(\mathrm{dd}, J=0.8,0.8 \mathrm{~Hz}, 1 \mathrm{H}), 7.59-7.56(\mathrm{~m}, 1 \mathrm{H}), 7.52-$ $7.48(\mathrm{~m}, 2 \mathrm{H}), 7.45-7.37(\mathrm{~m}, 3 \mathrm{H}), 2.55(\mathrm{~s}, 3 \mathrm{H}) \mathrm{ppm}$. The spectral data match those previously reported. ${ }^{31}$

N-Phenyl-4-tert-butylphthalimide (1s). Prepared according to Method A starting from 4-tert-butylphthalic anhydride in $93 \%$ isolated yield (1.3 g). ${ }^{1} \mathrm{H}$ NMR (400 MHz, $\left.\mathrm{CDCl}_{3}\right): \delta=7.99$ (dd, $J=$ $2.0,0.8 \mathrm{~Hz}, 1 \mathrm{H}), 7.88(\mathrm{dd}, J=8.0,0.8 \mathrm{~Hz}, 1 \mathrm{H}), 7.81(\mathrm{dd}, J=8.0$, $2.0 \mathrm{~Hz}, 1 \mathrm{H}), 7.53-7.48(\mathrm{~m}, 2 \mathrm{H}), 7.44-7.38(\mathrm{~m}, 3 \mathrm{H}), 1.41(\mathrm{~s}, 9 \mathrm{H})$ $\mathrm{ppm}$. The spectral data match those previously reported. ${ }^{28}$

$N$-Phenyl-4-fluorophthalimide (1t). Prepared according to Method A starting from 4-fluorophthalic anhydride in $89 \%$ isolated yield $(1.07 \mathrm{~g}) .{ }^{1} \mathrm{H}$ NMR $\left(400 \mathrm{MHz}, \mathrm{CDCl}_{3}\right): \delta=7.96(\mathrm{dd}, J=8.4$, $4.4 \mathrm{~Hz}, 1 \mathrm{H}), 7.62(\mathrm{dd}, J=7.2,2.0 \mathrm{~Hz}, 1 \mathrm{H}), 7.54-7.47(\mathrm{~m}, 2 \mathrm{H}), 7.46-$ $7.39(\mathrm{~m}, 4 \mathrm{H}) \mathrm{ppm} .{ }^{19} \mathrm{~F}\left\{{ }^{1} \mathrm{H}\right\}$ NMR $\left(376 \mathrm{MHz}, \mathrm{CDCl}_{3}\right): \delta=-101.1$ $\mathrm{ppm}$. The spectral data match those previously reported. ${ }^{\mathbf{2 8}}$

$N$-Phenyl-4-nitrophthalimide $(\mathbf{1} \boldsymbol{u})$. Prepared according to Method A starting from 4-nitrophthalic anhydride in 98\% isolated yield $(1.85 \mathrm{~g}) .{ }^{1} \mathrm{H}$ NMR $\left(400 \mathrm{MHz}, \mathrm{CDCl}_{3}\right): \delta=8.77$ (dd, $J=6.0$, $0.8 \mathrm{~Hz}, 1 \mathrm{H}), 8.67(\mathrm{dd}, J=8.0,2.0 \mathrm{~Hz}, 1 \mathrm{H}), 8.16(\mathrm{dd}, J=8.0,0.8$ $\mathrm{Hz}, 1 \mathrm{H}), 7.56-7.52(\mathrm{~m}, 2 \mathrm{H}), 7.48-7.43(\mathrm{~m}, 3 \mathrm{H}) \mathrm{ppm}$. The spectral data match those previously reported..$^{32}$

2-Phenylhexahydro-1H-isoindole-1,3(2H)-dione (1v). Prepared according to Method D in $86 \%$ isolated yield $(1.69 \mathrm{~g}) .{ }^{1} \mathrm{H}$ NMR $\left(400 \mathrm{MHz}, \mathrm{CDCl}_{3}\right): \delta=7.48-7.44(\mathrm{~m}, 2 \mathrm{H}), 7.39-7.35(\mathrm{~m}$, $1 \mathrm{H}), 7.30-7.27(\mathrm{~m}, 2 \mathrm{H}), 3.06-3.00(\mathrm{~m}, 2 \mathrm{H}), 1.95-1.85(\mathrm{~m}, 4 \mathrm{H})$, $1.53-1.50(\mathrm{~m}, 4 \mathrm{H}) \mathrm{ppm}$. The spectral data match those previously reported. ${ }^{33}$

$N$-Phenylsuccinimide (1w). Prepared according to Method E starting from succinic acid in $10 \%$ isolated yield $(35.0 \mathrm{mg}) .{ }^{1} \mathrm{H}$ NMR (400 MHz, $\left.\mathrm{CDCl}_{3}\right): \delta=7.50-7.45(\mathrm{~m}, 2 \mathrm{H}), 7.41-7.37$ (m, $1 \mathrm{H}), 7.29-7.26(\mathrm{~m}, 2 \mathrm{H}), 2.87(\mathrm{~s}, 4 \mathrm{H}) \mathrm{ppm}$. The spectral data match those previously reported. ${ }^{34}$

3-Methyl-1-phenyl-1H-pyrrole-2,5-dione (1x). Prepared according to Method A starting from citraconic anhydride in $77 \%$ isolated yield (1.15 g). ${ }^{1} \mathrm{H}$ NMR (400 MHz, $\left.\mathrm{CDCl}_{3}\right): \delta=7.48-7.43(\mathrm{~m}$, $2 \mathrm{H}), 7.36-7.32(\mathrm{~m}, 3 \mathrm{H}), 6.46(\mathrm{q}, J=2.0 \mathrm{~Hz}, 1 \mathrm{H}), 2.15(\mathrm{~d}, J=2.0$ $\mathrm{Hz}, 3 \mathrm{H}) \mathrm{ppm}$. The spectral data match those previously reported. ${ }^{35}$

2-Phenyl-1H-benzo[de]isoquinoline-1,3(2H)-dione (1y). Prepared according to Method A starting from 1,8-naphthalic anhydride in $78 \%$ isolated yield $(1.49 \mathrm{~g}) .{ }^{1} \mathrm{H}$ NMR (400 $\left.\mathrm{MHz}, \mathrm{CDCl}_{3}\right)$ : $\delta=8.64(\mathrm{dd}, J=7.2,1.2 \mathrm{~Hz}, 2 \mathrm{H}), 8.26(\mathrm{dd}, J=8.4,0.8 \mathrm{~Hz}, 2 \mathrm{H})$, $7.78(\mathrm{dd}, J=8.0,7.2 \mathrm{~Hz}, 2 \mathrm{H}), 7.59-7.54(\mathrm{~m}, 2 \mathrm{H}), 7.51-7.47(\mathrm{~m}$, $1 \mathrm{H}), 7.34(\mathrm{dd}, J=4.0,1.2 \mathrm{~Hz}, 2 \mathrm{H}) \mathrm{ppm}$. The spectral data match those previously reported. ${ }^{32}$

2-Phenylisoindolin-1-one (1z). Prepared according to the previous literature ${ }^{36}$ in $78 \%$ isolated yield $(1.4 \mathrm{~g}) .{ }^{1} \mathrm{H} \mathrm{NMR}(400 \mathrm{MHz}$, $\left.\mathrm{CDCl}_{3}\right): \delta=7.93(\mathrm{~d}, J=7.6 \mathrm{~Hz}, 1 \mathrm{H}), 7.89-7.86(\mathrm{~m}, 2 \mathrm{H}), 7.62-7.58$ (m, 1H), 7.53-7.49 (m, 2H), 7.43 (dd, $J=8.4,7.2 \mathrm{~Hz}, 2 \mathrm{H}), 7.18$ (dd, $J=7.2,7.2 \mathrm{~Hz}, 1 \mathrm{H}), 4.87$ (s, 2H) ppm. The spectral data match those previously reported. ${ }^{37}$

2-Phenyl-2,3-dihydro-1H-isoindole (1aa). Prepared according to Method C starting from 1,2-bis(bromomethyl)benzene in $89 \%$ isolated yield $(869 \mathrm{mg}) .{ }^{1} \mathrm{H} \mathrm{NMR}\left(400 \mathrm{MHz}, \mathrm{CDCl}_{3}\right): \delta=7.38-7.31$ $(\mathrm{m}, 6 \mathrm{H}), 6.78$ (dd, $J=7.6,7.6 \mathrm{~Hz}, 1 \mathrm{H}), 6.71(\mathrm{~d}, J=7.6 \mathrm{~Hz}, 2 \mathrm{H})$, $4.68(\mathrm{~s}, 4 \mathrm{H}) \mathrm{ppm}$. The spectral data match those previously reported. ${ }^{38}$ 
$N$-(p-Methoxyphenyl)phthalimide (1ab). Prepared according to Method A starting from $p$-anisidine in $80 \%$ isolated yield $(1.01 \mathrm{~g})$. ${ }^{1} \mathrm{H}$ NMR (400 MHz, $\left.\mathrm{CDCl}_{3}\right): \delta=7.94(\mathrm{dd}, J=5.2,2.8 \mathrm{~Hz}, 2 \mathrm{H})$, $7.78(\mathrm{dd}, J=5.6,3.2 \mathrm{~Hz}, 2 \mathrm{H}), 7.34(\mathrm{~d}, J=9.2 \mathrm{~Hz}, 2 \mathrm{H}), 7.02(\mathrm{~d}, J$ $=9.2 \mathrm{~Hz}, 2 \mathrm{H}), 3.85(\mathrm{~s}, 3 \mathrm{H}) \mathrm{ppm}$. The spectral data match those previously reported. ${ }^{\mathbf{1 7}, \mathbf{2 4}}$

p-Phthalimidoacetophenone (1ac). Prepared according to Method A starting from 4-aminoacetophenone in $79 \%$ isolated yield $(1.67 \mathrm{~g}) .{ }^{1} \mathrm{H}$ NMR (400 MHz, $\left.\mathrm{CDCl}_{3}\right): \delta=8.10(\mathrm{dd}, J=6.8$, $2.0 \mathrm{~Hz}, 2 \mathrm{H}), 7.97(\mathrm{dd}, J=5.6,3.2 \mathrm{~Hz}, 2 \mathrm{H}), 7.82(\mathrm{dd}, J=5.6,3,2$ $\mathrm{Hz}, 2 \mathrm{H}), 7.63(\mathrm{dd}, J=6.8,2.0 \mathrm{~Hz}, 2 \mathrm{H}), 2.64(\mathrm{~s}, 3 \mathrm{H}) \mathrm{ppm}$. The spectral data match those previously reported. ${ }^{17}$

$N$-(p-Cyanophenyl)phthalimide (1ad). Prepared according to Method A starting from 4-aminobenzonitrile in $93 \%$ isolated yield $(2.3 \mathrm{~g}) .{ }^{1} \mathrm{H}$ NMR $\left(400 \mathrm{MHz}, \mathrm{CDCl}_{3}\right): \delta=7.99(\mathrm{dd}, J=5.6,3.2 \mathrm{~Hz}$, $2 \mathrm{H}), 7.84(\mathrm{dd}, J=5.6,3.2 \mathrm{~Hz}, 2 \mathrm{H}), 7.80(\mathrm{dd}, J=6.4,2.0 \mathrm{~Hz}, 2 \mathrm{H})$, $7.69(\mathrm{dd}, J=6.4,2.0 \mathrm{~Hz}, 2 \mathrm{H}) \mathrm{ppm}$. The spectral data match those previously reported. ${ }^{39}$

$\mathrm{N}$-(o-Chlorophenyl)phthalimide (1ae). Prepared according to Method A starting from 2-chloroaniline in 70\% isolated yield (1.4 g). ${ }^{1} \mathrm{H}$ NMR $\left(400 \mathrm{MHz}, \mathrm{CDCl}_{3}\right): \delta=7.96(\mathrm{dd}, J=5.6,3.2 \mathrm{~Hz}, 2 \mathrm{H})$, $7.80(\mathrm{dd}, J=5.6,3.2 \mathrm{~Hz}, 2 \mathrm{H}), 7.59-7.55(\mathrm{~m}, 1 \mathrm{H}), 7.46-7.38(\mathrm{~m}$, $2 \mathrm{H}), 7.37-7.35(\mathrm{~m}, 1 \mathrm{H}) \mathrm{ppm}$. The spectral data match those previously reported. ${ }^{\mathbf{4 0}}$

$N$-(2-Biphenyl)phthalimide (1af). Prepared according to Method A starting from 2-chloroaniline in 50\% isolated yield (878 $\mathrm{mg}) .{ }^{1} \mathrm{H}$ NMR $\left(400 \mathrm{MHz}, \mathrm{CDCl}_{3}\right): \delta=7.81(\mathrm{dd}, J=5.6,3.2 \mathrm{~Hz}$, $2 \mathrm{H}), 7.70(\mathrm{dd}, J=5.6,3.2 \mathrm{~Hz}, 2 \mathrm{H}), 7.55-7.48(\mathrm{~m}, 3 \mathrm{H}), 7.35-7.32$ $(\mathrm{m}, 1 \mathrm{H}), 7.29-7.20(\mathrm{~m}, 5 \mathrm{H}) \mathrm{ppm}$. The spectral data match those previously reported. $12 \mathrm{c}$

N-(4-pyridyl)phthalimide (1ag). Prepared according to Method A starting from 4-aminopyridine in $76 \%$ isolated yield $(1.19 \mathrm{~g}) .{ }^{1} \mathrm{H}$ NMR $\left(400 \mathrm{MHz}, \mathrm{CDCl}_{3}\right): \delta=8.75(\mathrm{dd}, J=4.8,1.6 \mathrm{~Hz}, 2 \mathrm{H}), 7.99$ $(\mathrm{dd}, J=5.6,3.2 \mathrm{~Hz}, 2 \mathrm{H}), 7.84(\mathrm{dd}, J=5.6,3.2 \mathrm{~Hz}, 2 \mathrm{H}), 7.61(\mathrm{dd}$, $J=4.8,1.6 \mathrm{~Hz}, 2 \mathrm{H}) \mathrm{ppm}$. The spectral data match those previously reported. ${ }^{41}$

General procedure for the ruthenium-catalysed hydroxylation reaction and characterization of products $(2-4)$. $\left[\mathrm{RuCl}_{2}(p-\right.$ cymene $)]_{2}(0.003 \mathrm{mmol}, 1.8 \mathrm{mg}, 0.01$ eq.), ammonium persulfate (0.36 mmol, $82.2 \mathrm{mg}, 1.2$ eq.), substrate 1 ( $0.3 \mathrm{mmol}, 1$ eq.) and TFA/TFAA $(1.0 \mathrm{~mL}, 3: 1, v / v)$ were introduced in a flame-dried Schlenk tube under air atmosphere. The reaction mixture was stirred at $80{ }^{\circ} \mathrm{C}$ during 15 hours. Then, the reaction mixture was cooled down to room temperature and diluted with $\mathrm{H}_{2} \mathrm{O}(50 \mathrm{~mL})$ followed by extraction with $\mathrm{CH}_{2} \mathrm{Cl}_{2}(3 \times 20 \mathrm{~mL})$. The combined organic layers were washed with brine $(30 \mathrm{~mL})$ and dried over $\mathrm{Na}_{2} \mathrm{SO}_{4}$. After filtration and evaporation of the solvents in vacuo, the crude product was purified by column chromatography on silica gel ( $n$-heptane/EtOAc: $4 / 1, v / v)$ to give product 2.

2-(2-Hydroxyphenyl)isoindoline-1,3-dione (2a). Yellow solid, yield $=82 \%, 60.9 \mathrm{mg} .{ }^{1} \mathrm{H}$ NMR $\left(400 \mathrm{MHz}, \mathrm{CDCl}_{3}\right): \delta=7.97(\mathrm{dd}$, $J=5.6,3.2 \mathrm{~Hz}, 2 \mathrm{H}), 7.81(\mathrm{dd}, J=5.6,3.2 \mathrm{~Hz}, 2 \mathrm{H}), 7.36-7.30(\mathrm{~m}$, 2H), 7.11-7.07 (m, 2H), 5.80 (br, 1H) ppm. ${ }^{13} \mathrm{C}\left\{{ }^{1} \mathrm{H}\right\}$ NMR $(100$ MHz, acetone- $\left.d_{6}\right): \delta=167.9,154.9,135.2,133.5,131.3,131.2$, 124.0, 120.6, 120.4, 117.5 ppm. MS (EI): $m / z=239\left(\mathrm{M}^{+}, 52\right), 195$ (100), 104 (39), 76 (64). The spectral data match those previously reported. ${ }^{42}$

2-(2-Hydroxy-4-methylphenyl)isoindoline-1,3-dione (2b). Colorless solid, yield $=90 \%, 68.4 \mathrm{mg} . \mathrm{Mp}>250{ }^{\circ} \mathrm{C} \mathrm{dec} .{ }^{1} \mathrm{H}$ NMR $(400$ $\left.\mathrm{MHz} \mathrm{CDCl}_{3}\right): \delta=7.96(\mathrm{dd}, J=5.6,3.2 \mathrm{~Hz}, 2 \mathrm{H}), 7.80(\mathrm{dd}, J=5.6$, $3.2 \mathrm{~Hz}, 2 \mathrm{H}), 7.18(\mathrm{~d}, J=7.6 \mathrm{~Hz}, 1 \mathrm{H}), 6.90(\mathrm{~d}, J=8.0 \mathrm{~Hz}, 2 \mathrm{H}), 5.71$ (br, $1 \mathrm{H}), 2.36(\mathrm{~s}, 3 \mathrm{H}) \mathrm{ppm} .{ }^{13} \mathrm{C}\left\{{ }^{1} \mathrm{H}\right\} \mathrm{NMR}\left(100 \mathrm{MHz}\right.$, acetone- $\left.d_{6}\right)$ : $\delta=168.1,154.6,141.4,135.1,133.5,130.9,124.0,121.4,118.0$, 117.7, 21.3 ppm. HRMS (ESI) calcd. for $[\mathrm{M}+\mathrm{Na}]^{+} \mathrm{C}_{15} \mathrm{H}_{11} \mathrm{NO}_{3} \mathrm{Na}$ 276.0631, found 276.0635 (1 ppm).
2-(4-Diethylamino-2-hydroxyphenyl)isoindoline-1,3-dione (2c). Brown oil, yield $=88 \%, 65.0 \mathrm{mg} .{ }^{1} \mathrm{H}$ NMR $(400 \mathrm{MHz}$, acetone- $\left.d_{6}\right): \delta=7.95-7.88(\mathrm{~m}, 4 \mathrm{H}), 7.31(\mathrm{~d}, J=8.4 \mathrm{~Hz}, 1 \mathrm{H}), 7.05(\mathrm{~d}$, $J=2.4 \mathrm{~Hz}, 1 \mathrm{H}), 6.99(\mathrm{dd}, J=8.4,2.4 \mathrm{~Hz}, 1 \mathrm{H}), 3.02(\mathrm{q}, J=7.2 \mathrm{~Hz}$, $4 \mathrm{H}), 0.99(\mathrm{t}, J=7.2 \mathrm{~Hz}, 6 \mathrm{H}) \mathrm{ppm} .{ }^{13} \mathrm{C}\left\{{ }^{1} \mathrm{H}\right\}$ NMR $(100 \mathrm{MHz}$, acetone- $\left.d_{6}\right): \delta=167.8,155.0,137.2,135.3,132.8,130.6,124.1,124.0$, 119.1, 113.6, 49.8, 13.0 ppm. HRMS (ESI) calcd. for $[\mathrm{M}+\mathrm{Na}]^{+}$ $\mathrm{C}_{18} \mathrm{H}_{18} \mathrm{~N}_{2} \mathrm{O}_{3} \mathrm{Na} 333.1210$, found 333.1213 (1 ppm).

2-(4-Fluoro-2-hydroxyphenyl)isoindoline-1,3-dione (2d). Colorless solid, yield $=78 \%, 45.5 \mathrm{mg}$. Mp: $225-228{ }^{\circ} \mathrm{C} .{ }^{1} \mathrm{H}$ NMR $(400$ $\mathrm{MHz}$, acetone- $\left.d_{6}\right): \delta=9.27(\mathrm{br}, 1 \mathrm{H}), 7.95-7.89(\mathrm{~m}, 4 \mathrm{H}), 7.37(\mathrm{dd}$ $J=8.8,6.4 \mathrm{~Hz}, 1 \mathrm{H}), 6.85-6.75(\mathrm{~m}, 2 \mathrm{H}) \mathrm{ppm} .{ }^{13} \mathrm{C}\left\{{ }^{1} \mathrm{H}\right\}$ NMR $(100$ $\mathrm{MHz}$, acetone- $\left.d_{6}\right): \delta=167.9,164.4\left(\mathrm{~d}, J_{\mathrm{C}-\mathrm{F}}=243.9 \mathrm{~Hz}\right), 156.3(\mathrm{~d}$, $\left.J_{\mathrm{C}-\mathrm{F}}=12.5 \mathrm{~Hz}\right), 135.2,133.4,132.6\left(\mathrm{~d}, J_{\mathrm{C}-\mathrm{F}}=10.8 \mathrm{~Hz}\right), 124.1,116.8$ $\left(\mathrm{d}, J_{\mathrm{C}-\mathrm{F}}=3.3 \mathrm{~Hz}\right), 107.4\left(\mathrm{~d}, J_{\mathrm{C}-\mathrm{F}}=22.9 \mathrm{~Hz}\right), 104.6\left(\mathrm{~d}, J_{\mathrm{C}-\mathrm{F}}=25.2\right.$ Hz) ppm. ${ }^{19} \mathrm{~F}\left\{{ }^{1} \mathrm{H}\right\}$ NMR (376 MHz, acetone- $\left.d_{6}\right): \delta=-112.3 \mathrm{ppm}$. HRMS (ESI) calcd. for $[\mathrm{M}+\mathrm{Na}]^{+} \mathrm{C}_{14} \mathrm{H}_{8} \mathrm{NO}_{3} \mathrm{FNa} 280.0380$, found 280.0381 (0 ppm).

2-(4-Chloro-2-hydroxyphenyl)isoindoline-1,3-dione (2e). Colorless solid, yield $=85 \%, 60.6 \mathrm{mg} .{ }^{1} \mathrm{H}$ NMR $(400 \mathrm{MHz}$, acetone$\left.d_{6}\right): \delta=9.24(\mathrm{br}, 1 \mathrm{H}), 7.96-7.90(\mathrm{~m}, 4 \mathrm{H}), 7.36(\mathrm{~d}, J=8.4 \mathrm{~Hz}, 1 \mathrm{H})$, $7.11(\mathrm{~d}, J=2.0 \mathrm{~Hz}, 1 \mathrm{H}), 7.03(\mathrm{dd}, J=8.4,2.0 \mathrm{~Hz}, 1 \mathrm{H}) \mathrm{ppm}$. ${ }^{13} \mathrm{C}\left\{{ }^{1} \mathrm{H}\right\}$ NMR $(100 \mathrm{MHz}$, acetone- $d 6): \delta=167.7,155.7,135.8$, $135.2,133.3,132.5,124.1,120.7,119.4,117.6 \mathrm{ppm}$. MS (EI): $\mathrm{m} / \mathrm{z}$ $=273\left(\mathrm{M}^{+}, 44\right), 229(100), 104$ (46), 76 (78). The spectral data match those previously reported. ${ }^{42}$

2-(4-Bromo-2-hydroxyphenyl)isoindoline-1,3-dione (2f). Colorless solid, yield $=87 \%, 83.0 \mathrm{mg}$. ${ }^{1} \mathrm{H}$ NMR $(400 \mathrm{MHz}$, acetone$\left.d_{6}\right): \delta=7.95-7.89(\mathrm{~m}, 4 \mathrm{H}), 7.29(\mathrm{~d}, J=8.4 \mathrm{~Hz}, 1 \mathrm{H}), 7.26(\mathrm{~d}, J=2.0$ $\mathrm{Hz}, 1 \mathrm{H}), 7.18(\mathrm{dd}, J=8.4,2.0 \mathrm{~Hz}, 1 \mathrm{H}) \mathrm{ppm} .{ }^{13} \mathrm{C}\left\{{ }^{1} \mathrm{H}\right\} \mathrm{NMR}(100$ $\mathrm{MHz}$, acetone- $\left.d_{6}\right): \delta=167.6,155.9,135.2,133.3,132.8,124.1$, $123.70,123.69,120.5,119.9 \mathrm{ppm}$. MS (EI): $m / z=317\left(\mathrm{M}^{+}, 52\right)$, 275 (94), 104 (61), 76 (100), 50 (35). The spectral data match those previously reported. $\mathbf{4 2}^{2}$

2-(2-Hydroxy-4-iodophenyl)isoindoline-1,3-dione (2g). Yellow solid, yield $=88 \%, 96.3 \mathrm{mg}$. Mp: $184-187{ }^{\circ} \mathrm{C}$. ${ }^{1} \mathrm{H}$ NMR $(400$ MHz, acetone- $\left.d_{6}\right): \delta=9.15(\mathrm{br}, 1 \mathrm{H}), 7.95-7.89(\mathrm{~m}, 4 \mathrm{H}), 7.46(\mathrm{~d}, J$ $=2.0 \mathrm{~Hz}, 1 \mathrm{H}), 7.37(\mathrm{dd}, J=8.0,2.0 \mathrm{~Hz}, 1 \mathrm{H}), 7.13(\mathrm{~d}, J=8.0 \mathrm{~Hz}$, 1H) ppm. ${ }^{13} \mathrm{C}\left\{{ }^{1} \mathrm{H}\right\}$ NMR $\left(100 \mathrm{MHz}\right.$, acetone- $\left.d_{6}\right): \delta=167.6,155.7$, 135.2, 133.3, 133.0, 129.9, 126.6, 124.1, 120.6, 95.3 ppm. HRMS (ESI) calcd. for $[\mathrm{M}+\mathrm{Na}]^{+} \mathrm{C}_{14} \mathrm{H}_{8} \mathrm{NO}_{3} \mathrm{INa} 387.9441$, found 387.9445 (1 ppm).

2-(4-Ethoxycarbonyl-2-hydroxyphenyl)isoindoline-1,3-dione (2h). Colorless solid, yield $=81 \%, 47.8 \mathrm{mg}$. Mp: $210-213{ }^{\circ} \mathrm{C} .{ }^{1} \mathrm{H}$ NMR (400 MHz, acetone- $d 6): \delta=7.97-7.91(\mathrm{~m}, 4 \mathrm{H}), 7.71(\mathrm{~d}, J=$ $2.0 \mathrm{~Hz}, 1 \mathrm{H}), 7.64(\mathrm{dd}, J=8.0,2.0 \mathrm{~Hz}, 1 \mathrm{H}), 7.47(\mathrm{~d}, J=8.0 \mathrm{~Hz}, 1 \mathrm{H})$, $4.37(\mathrm{q}, J=7.2 \mathrm{~Hz}, 2 \mathrm{H}), 1.38(\mathrm{t}, J=7.2 \mathrm{~Hz}, 3 \mathrm{H}) \mathrm{ppm} .{ }^{13} \mathrm{C}\left\{{ }^{1} \mathrm{H}\right\}$ NMR (100 MHz, acetone- $d 6): \delta=167.5,166.1,154.8,135.3$, $133.40,133.37,131.5,124.7,124.2,121.4,118.2,61.7,14.5 \mathrm{ppm}$. HRMS (ESI) calcd. for $[\mathrm{M}+\mathrm{Na}]^{+} \mathrm{C}_{17} \mathrm{H}_{13} \mathrm{NO}_{5} \mathrm{Na} 334.0686$, found 334.0686 (0 ppm).

2-(2-Hydroxy-4-nitrophenyl)isoindoline-1,3-dione (2i). Colorless solid, yield $=53 \%, 45.2 \mathrm{mg} .{ }^{1} \mathrm{H}$ NMR $\left(400 \mathrm{MHz}, \mathrm{DMSO}-d_{6}\right)$ : $\delta=11.01(\mathrm{br}, 1 \mathrm{H}), 8.01-7.92(\mathrm{~m}, 4 \mathrm{H}), 7.82-7.78(\mathrm{~m}, 2 \mathrm{H}), 7.62(\mathrm{~d}$, $J=8.0 \mathrm{~Hz}, 1 \mathrm{H}) \mathrm{ppm} .{ }^{13} \mathrm{C}\left\{{ }^{1} \mathrm{H}\right\}$ NMR $\left(100 \mathrm{MHz}, \mathrm{DMSO}-d_{6}\right): \delta=$ $166.3,154.7,148.4,134.8,131.8,131.5,125.3,123.5,113.9,111.1$ ppm. MS (EI): $m / z=248\left(\mathrm{M}^{+}, 100\right), 204$ (85), 104 (30), 76 (85), 50 (31). The spectral data match those previously reported. ${ }^{43}$

2-(5-Bromo-2-hydroxyphenyl)isoindoline-1,3-dione (2j). Colorless solid, yield $=87 \%, 71.5 \mathrm{mg} .{ }^{1} \mathrm{H}$ NMR $(400 \mathrm{MHz}$, acetone$\left.d_{6}\right): \delta=9.08(\mathrm{br}, 1 \mathrm{H}), 7.96-7.90(\mathrm{~m}, 4 \mathrm{H}), 7.57(\mathrm{~d}, J=2.4 \mathrm{~Hz}, 1 \mathrm{H})$, 7.50 (dd, $J=8.8,2.4 \mathrm{~Hz}, 1 \mathrm{H}), 7.05(\mathrm{~d}, J=8.8 \mathrm{~Hz}, 1 \mathrm{H}) \mathrm{ppm}$. ${ }^{13} \mathrm{C}\left\{{ }^{1} \mathrm{H}\right\}$ NMR $\left(100 \mathrm{MHz}\right.$, acetone- $\left.d_{6}\right): \delta=167.6,154.5,135.3$, 134.0, 133.8, 133.3, 124.1, 121.9, 119.4, $111.0 \mathrm{ppm}$. MS (EI): $\mathrm{m} / \mathrm{z}$ $=317\left(\mathrm{M}^{+}, 34\right), 273(82), 104$ (60), 76 (100), 50 (34). The spectral data match those previously reported. ${ }^{42}$ 
2-(2-Hydroxy-5-iodophenyl)isoindoline-1,3-dione (2k). Brown solid, yield $=85 \%, 93.0 \mathrm{mg}$. Mp $>250{ }^{\circ} \mathrm{C} \mathrm{dec} .{ }^{1} \mathrm{H}$ NMR $(400 \mathrm{MHz}$, acetone- $\left.d^{6}\right): \delta=9.10(\mathrm{br}, 1 \mathrm{H}), 7.96-7.90(\mathrm{~m}, 4 \mathrm{H}), 7.71(\mathrm{~d}, J=2.4$ $\mathrm{Hz}, 1 \mathrm{H}), 7.66(\mathrm{dd}, J=8.8,2.4 \mathrm{~Hz}, 1 \mathrm{H}), 6.92(\mathrm{~d}, J=8.8 \mathrm{~Hz}, 1 \mathrm{H})$ ppm. ${ }^{13} \mathrm{C}\left\{{ }^{1} \mathrm{H}\right\}$ NMR (100 MHz, acetone- $\left.d_{6}\right): \delta=167.6,155.2$, 140.0, 139.7, 135.3, 133.4, 124.1, 122.2, 119.9, 80.2 ppm. HRMS (ESI) calcd. for $[\mathrm{M}+\mathrm{Na}]^{+} \mathrm{C}_{14} \mathrm{H}_{8} \mathrm{NO}_{3} \mathrm{INa} 387.9441$, found 387.9443 (0 ppm).

2-(6-Fluoro-2-hydroxyphenyl)isoindoline-1,3-dione (2l). Yellow solid, yield $=42 \%$, $32.4 \mathrm{mg}$. Mp: $204-207{ }^{\circ} \mathrm{C} .{ }^{1} \mathrm{H}$ NMR $(400$ $\mathrm{MHz}$, acetone- $\left.d_{6}\right): \delta=9.31(\mathrm{br}, 1 \mathrm{H}), 8.01-7.94(\mathrm{~m}, 4 \mathrm{H}), 7.39(\mathrm{dd}$, $J=8.4,6.8 \mathrm{~Hz}, 1 \mathrm{H}), 6.92(\mathrm{~d}, J=8.4 \mathrm{~Hz}, 1 \mathrm{H}), 6.84(\mathrm{dd}, J=8.4,8.4$ $\mathrm{Hz}, 1 \mathrm{H}) \mathrm{ppm} .{ }^{13} \mathrm{C}\left\{{ }^{1} \mathrm{H}\right\}$ NMR $\left(100 \mathrm{MHz}\right.$, acetone- $\left.d_{6}\right): \delta=167.2$, $160.6\left(\mathrm{~d}, J_{\mathrm{C}-\mathrm{F}}=246.5 \mathrm{~Hz}\right), 156.9\left(\mathrm{~d}, J_{\mathrm{C}-\mathrm{F}}=3.6 \mathrm{~Hz}\right), 135.6,133.2$, $131.9\left(\mathrm{~d}, J_{\mathrm{C}-\mathrm{F}}=10.5 \mathrm{~Hz}\right), 124.4,113.3\left(\mathrm{~d}, J_{\mathrm{C}-\mathrm{F}}=3.0 \mathrm{~Hz}\right), 108.8(\mathrm{~d}$, $\left.J_{\mathrm{C}-\mathrm{F}}=16.0 \mathrm{~Hz}\right), 107.3\left(\mathrm{~d}, J_{\mathrm{C}-\mathrm{F}}=20.0 \mathrm{~Hz}\right) \mathrm{ppm} .{ }^{19} \mathrm{~F}\left\{{ }^{1} \mathrm{H}\right\} \mathrm{NMR}(376$ $\mathrm{MHz}$, acetone- $d_{6}$ ): $\delta=-122.0 \mathrm{ppm}$. HRMS (ESI) calcd. for $[\mathrm{M}+\mathrm{Na}]^{+} \mathrm{C}_{14} \mathrm{H}_{8} \mathrm{NO}_{3} \mathrm{FNa} 280.0380$, found 280.0383 (1 ppm).

2-(2-Hydroxy-6-methylphenyl)isoindoline-1,3-dione (2m). Colorless solid, yield $=28 \%, 21.1 \mathrm{mg}$. Mp: $145-148{ }^{\circ} \mathrm{C} .{ }^{1} \mathrm{H}$ NMR (400 MHz, acetone- $\left.d_{6}\right): \delta=8.75(\mathrm{br}, 1 \mathrm{H}), 7.97-7.91(\mathrm{~m}, 4 \mathrm{H}), 7.22$ (dd, $J=8.0,8.0 \mathrm{~Hz}, 1 \mathrm{H}), 6.88(\mathrm{dd}, J=8.0,8.0 \mathrm{~Hz}, 2 \mathrm{H}), 2.14(\mathrm{~s}$, $3 \mathrm{H}) \mathrm{ppm} .{ }^{13} \mathrm{C}\left\{{ }^{1} \mathrm{H}\right\} \mathrm{NMR}\left(100 \mathrm{MHz}\right.$, acetone- $\left.d_{6}\right): \delta=167.9,155.1$, 139.4, 135.3, 133.4, 130.8, 124.1, 122.0, 119.7, 114.8, 17.9 ppm. HRMS (ESI) calcd. for $[\mathrm{M}+\mathrm{Na}]^{+} \mathrm{C}_{15} \mathrm{H}_{11} \mathrm{NO}_{3} \mathrm{Na} 276.0631$, found 276.0635 (1 ppm).

2-(2-Hydroxyphenyl)-4-methylisoindoline-1,3-dione (2n). Colorless solid, yield $=90 \%, 68.3 \mathrm{mg}$. Mp: $187-190{ }^{\circ} \mathrm{C}$. $1 \mathrm{H}$ NMR $(400$ MHz, acetone- $\left.d_{6}\right): \delta=8.77(\mathrm{br}, 1 \mathrm{H}), 7.74-7.72(\mathrm{~m}, 2 \mathrm{H}), 7.65-7.63$ $(\mathrm{m}, 1 \mathrm{H}), 7.35-7.29(\mathrm{~m}, 2 \mathrm{H}), 7.07(\mathrm{dd}, J=8.0,1.2 \mathrm{~Hz}, 1 \mathrm{H}), 6.98$ (ddd, $J=7.6,7.6,1.2 \mathrm{~Hz}, 1 \mathrm{H}), 2.70(\mathrm{~s}, 3 \mathrm{H}) \mathrm{ppm} .{ }^{13} \mathrm{C}\left\{{ }^{1} \mathrm{H}\right\} \mathrm{NMR}$ (100 MHz, acetone- $\left.d_{6}\right): \delta=168.7,167.8,154.9,138.6,137.2$, 134.6, 133.8, 131.3, 131.1, 129.9, 121.6, 120.5, 120.4, 117.5, 17.6 ppm. HRMS (ESI) calcd. for $[\mathrm{M}+\mathrm{Na}]^{+} \mathrm{C}_{15} \mathrm{H}_{11} \mathrm{NO}_{3} \mathrm{Na} 276.0631$, found 276.0634 (1 ppm).

4-Fluoro-2-(2-hydroxyphenyl)isoindoline-1,3-dione (2o). Colorless solid, yield $=84 \%, 64.7 \mathrm{mg}$. Mp: $200-203^{\circ} \mathrm{C}$. $1 \mathrm{H}$ NMR $(400$ MHz, acetone- $\left.d_{6}\right): \delta=8.82(\mathrm{br}, 1 \mathrm{H}), 7.95(\mathrm{ddd}, J=8.0,8.0,4.4 \mathrm{~Hz}$, $1 \mathrm{H}), 7.79(\mathrm{~d}, J=7.2 \mathrm{~Hz}, 1 \mathrm{H}), 7.63(\mathrm{dd}, J=8.8,8.8 \mathrm{~Hz}, 1 \mathrm{H}), 7.37$ $7.32(\mathrm{~m}, 2 \mathrm{H}), 7.07(\mathrm{dd}, J=8.0,1.2 \mathrm{~Hz}, 1 \mathrm{H}), 6.99(\mathrm{ddd}, J=7.6,7.6$, $1.2 \mathrm{~Hz}, 1 \mathrm{H}) \mathrm{ppm} .{ }^{13} \mathrm{C}\left\{{ }^{1} \mathrm{H}\right\} \mathrm{NMR}\left(100 \mathrm{MHz}\right.$, acetone- $\left.d_{6}\right): \delta=166.8$ $\left(\mathrm{d}, J_{\mathrm{C}-\mathrm{F}}=3.1 \mathrm{~Hz}\right), 164.6\left(\mathrm{~d}, J_{\mathrm{C}-\mathrm{F}}=1.6 \mathrm{~Hz}\right), 158.4\left(\mathrm{~d}, J_{\mathrm{C}-\mathrm{F}}=261.1\right.$ $\mathrm{Hz}), 154.8,138.1\left(\mathrm{~d}, J_{\mathrm{C}-\mathrm{F}}=7.7 \mathrm{~Hz}\right), 135.7\left(\mathrm{~d}, J_{\mathrm{C}-\mathrm{F}}=1.8 \mathrm{~Hz}\right), 131.3$ $\left(\mathrm{d}, J_{\mathrm{C}-\mathrm{F}}=18.7 \mathrm{~Hz}\right), 123.2\left(\mathrm{~d}, J_{\mathrm{C}-\mathrm{F}}=19.8 \mathrm{~Hz}\right), 120.6,120.4\left(\mathrm{~d}, J_{\mathrm{C}-\mathrm{F}}\right.$ $=3.7 \mathrm{~Hz}), 120.0,119.0\left(\mathrm{~d}, J_{\mathrm{C}-\mathrm{F}}=12.5 \mathrm{~Hz}\right), 117.6 \mathrm{ppm} .{ }^{19} \mathrm{~F}\left\{{ }^{1} \mathrm{H}\right\}$ NMR (376 MHz, acetone- $\left.d_{6}\right): \delta=-115.7 \mathrm{ppm}$. HRMS (ESI) calcd. for $[\mathrm{M}+\mathrm{Na}]^{+} \mathrm{C}_{14} \mathrm{H}_{8} \mathrm{NO}_{3} \mathrm{FNa} 280.0380$, found 280.0383 (1 ppm).

4-Chloro-2-(2-hydroxyphenyl)isoindoline-1,3-dione (2p). Colorless solid, yield $=71 \%, 58.1 \mathrm{mg}$. Mp: $209-212^{\circ} \mathrm{C}$. $1 \mathrm{H}$ NMR $(400$ $\mathrm{MHz}$, acetone- $\left.d_{6}\right): \delta=8.82(\mathrm{br}, 1 \mathrm{H}), 7.91-7.84(\mathrm{~m}, 3 \mathrm{H}), 7.37-7.32$ (m, 2H), $7.07(\mathrm{dd}, J=8.0,1.2 \mathrm{~Hz}, 1 \mathrm{H}), 6.99(\mathrm{ddd}, J=7.6,7.6,1.2$ $\mathrm{Hz}, 1 \mathrm{H})$ ppm. ${ }^{13} \mathrm{C}\left\{{ }^{1} \mathrm{H}\right\}$ NMR $\left(100 \mathrm{MHz}\right.$, acetone- $\left.d_{6}\right): \delta=166.5$, $165.5,154.9,136.6,136.5,135.6,131.39,131.36,131.2,129.0$, 122.8, 120.6, 120.0, 117.6 ppm. HRMS (ESI) calcd. for $[\mathrm{M}+\mathrm{Na}]^{+}$ $\mathrm{C}_{14} \mathrm{H}_{8} \mathrm{NO}_{3} \mathrm{ClNa} 296.0085$, found 296.0086 (0 ppm).

2-(2-Hydroxyphenyl)-4-nitroisoindoline-1,3-dione (2q). Colorless solid, yield $=76 \%, 64.8 \mathrm{mg}$. Mp: $226-229^{\circ} \mathrm{C}$. ${ }^{1} \mathrm{H}$ NMR $(400$ MHz, acetone- $\left.d_{6}\right): \delta=8.87(\mathrm{br}, 1 \mathrm{H}), 8.30-8.25(\mathrm{~m}, 2 \mathrm{H}), 8.18(\mathrm{dd}$, $J=7.6,7.6 \mathrm{~Hz}, 1 \mathrm{H}), 7.38-7.34(\mathrm{~m}, 2 \mathrm{H}), 7.08$ (ddd, $J=7.6,1.2,1.2$ $\mathrm{Hz}, 1 \mathrm{H}), 6.99$ (ddd, $J=7.6,7.6,1.2 \mathrm{~Hz}, 1 \mathrm{H}) \mathrm{ppm} .{ }^{13} \mathrm{C}\left\{{ }^{1} \mathrm{H}\right\} \mathrm{NMR}$ $\left(100 \mathrm{MHz}\right.$, acetone- $\left.d_{6}\right): \delta=165.9,163.3,154.8,146.1,137.1$, 135.2, 131.6, 131.1, 129.1, 127.6, 124.4, 120.7, 119.7, 117.7 ppm. HRMS (ESI) calcd. for $[\mathrm{M}+\mathrm{Na}]^{+} \mathrm{C}_{14} \mathrm{H}_{8} \mathrm{~N}_{2} \mathrm{O}_{5} \mathrm{Na} 307.0325$, found 307.0327 (0 ppm).

2-(2-Hydroxyphenyl)-5-methylisoindoline-1,3-dione (2r). Colorless solid, yield =93\%, $70.6 \mathrm{mg}$. Mp: $216-219^{\circ} \mathrm{C}$. $1 \mathrm{H}$ NMR (400
MHz, acetone- $\left.d_{6}\right): \delta=8.71(\mathrm{br}, 1 \mathrm{H}), 7.81(\mathrm{~d}, J=7.6 \mathrm{~Hz}, 1 \mathrm{H}), 7.75$ $(\mathrm{s}, 1 \mathrm{H}), 7.71(\mathrm{~d}, J=7.6 \mathrm{~Hz}, 1 \mathrm{H}), 7.35-7.27(\mathrm{~m}, 2 \mathrm{H}), 7.05(\mathrm{dd}, J=$ 8.0, 1.2 Hz, 1H), 6.97 (ddd, $J=7.6,7.6,1.2 \mathrm{~Hz}, 1 \mathrm{H}), 2.56(\mathrm{~s}, 3 \mathrm{H})$ ppm. ${ }^{13} \mathrm{C}\left\{{ }^{1} \mathrm{H}\right\}$ NMR $\left(100 \mathrm{MHz}\right.$, acetone- $\left.d_{6}\right): \delta=168.0,167.9$, $154.9,146.5,135.6,133.8,131.3,131.1,130.9,124.4,123.9$, $120.6,117.5,21.8 \mathrm{ppm}$. HRMS (ESI) calcd. for $[\mathrm{M}+\mathrm{Na}]^{+}$ $\mathrm{C}_{15} \mathrm{H}_{11} \mathrm{NO}_{3} \mathrm{Na} 276.0631$, found 276.0632 (0 ppm).

5-(tert-Butyl)-2-(2-hydroxyphenyl)isoindoline-1,3-dione (2s). Colorless solid, yield $=87 \%, 77.0 \mathrm{mg}$. Mp: $116-119{ }^{\circ} \mathrm{C} .{ }^{1} \mathrm{H}$ NMR (400 MHz, acetone- $\left.d_{6}\right): \delta=7.96-7.95(\mathrm{~m}, 2 \mathrm{H}), 7.86(\mathrm{~d}, J=8.4 \mathrm{~Hz}$, $1 \mathrm{H}), 7.36-7.29(\mathrm{~m}, 2 \mathrm{H}), 7.07$ (dd, $J=8.4,1.2 \mathrm{~Hz}, 1 \mathrm{H}), 6.98$ (ddd, $J=8.4,8.4,1.2 \mathrm{~Hz}, 1 \mathrm{H}), 1.44(\mathrm{~s}, 9 \mathrm{H}) \mathrm{ppm} .{ }^{13} \mathrm{C}\left\{{ }^{1} \mathrm{H}\right\} \mathrm{NMR}(100$ MHz, acetone- $\left.d_{6}\right): \delta=168.2,167.8,159.4,154.9,133.6,132.1$, $131.2,131.1,130.8,123.9,121.0,120.54,120.47,117.5,36.3,31.4$ ppm. HRMS (ESI) calcd. for $[\mathrm{M}+\mathrm{Na}]^{+} \mathrm{C}_{18} \mathrm{H}_{17} \mathrm{NO}_{3} \mathrm{Na} 318.1101$, found 318.1104 (1 ppm).

5-Fuloro-2-(2-hydroxyphenyl)isoindoline-1,3-dione (2t). Colorless solid, yield $=91 \%, 70.2 \mathrm{mg} . \mathrm{Mp}: 190-193^{\circ} \mathrm{C}$. ${ }^{1} \mathrm{H}$ NMR $(400$ MHz, acetone- $\left.d_{6}\right): \delta=8.00(\mathrm{dd}, J=8.4,4.4 \mathrm{~Hz}, 1 \mathrm{H}), 7.71-7.63(\mathrm{~m}$, 2H), 7.36-7.30 (m, 2H), 7.07 (dd, $J=8.0,1.2 \mathrm{~Hz}, 1 \mathrm{H}), 6.98$ (ddd, $J=7.6,7.6,1.2 \mathrm{~Hz}, 1 \mathrm{H}) \mathrm{ppm} .{ }^{13} \mathrm{C}\left\{{ }^{1} \mathrm{H}\right\}$ NMR $(100 \mathrm{MHz}$, acetone$\left.d_{6}\right): \delta=167.2\left(\mathrm{~d}, J_{\mathrm{C}-\mathrm{F}}=252.5 \mathrm{~Hz}\right), 166.9,166.6\left(\mathrm{~d}, J_{\mathrm{C}-\mathrm{F}}=2.9 \mathrm{~Hz}\right)$, $154.8,136.3\left(\mathrm{~d}, J_{\mathrm{C}-\mathrm{F}}=9.5 \mathrm{~Hz}\right), 131.3\left(\mathrm{~d}, J_{\mathrm{C}-\mathrm{F}}=17.7 \mathrm{~Hz}\right), 129.4(\mathrm{~d}$, $\left.J_{\mathrm{C}-\mathrm{F}}=2.8 \mathrm{~Hz}\right), 126.8\left(\mathrm{~d}, J_{\mathrm{C}-\mathrm{F}}=9.5 \mathrm{~Hz}\right), 122.0\left(\mathrm{~d}, J_{\mathrm{C}-\mathrm{F}}=23.8 \mathrm{~Hz}\right)$, 120.6, 120.2, 117.5, $111.6\left(\mathrm{~d}, J_{\mathrm{C}-\mathrm{F}}=25.0 \mathrm{~Hz}\right) \mathrm{ppm} .{ }^{19} \mathrm{~F}\left\{{ }^{1} \mathrm{H}\right\} \mathrm{NMR}$ (376 MHz, acetone- $d_{6}$ ): $\delta=-104.5 \mathrm{ppm}$. HRMS (ESI) calcd. for $[\mathrm{M}+\mathrm{Na}]^{+} \mathrm{C}_{14} \mathrm{H}_{8} \mathrm{NO}_{3} \mathrm{FNa} 280.0380$, found 280.0382 (1 ppm).

2-(2-Hydroxyphenyl)-5-nitroisoindoline-1,3-dione $(2 \mathrm{u})$. Yellow solid, yield $=82 \%, 69.9 \mathrm{mg} .{ }^{1} \mathrm{H}$ NMR $\left(400 \mathrm{MHz}\right.$, acetone- $\left.d_{6}\right)$ : $\delta=8.96(\mathrm{br}, 1 \mathrm{H}), 8.76(\mathrm{dd}, J=8.4,2.0 \mathrm{~Hz}, 1 \mathrm{H}), 8.64(\mathrm{~d}, J=2.0$ $\mathrm{Hz}, 1 \mathrm{H}), 8.23(\mathrm{~d}, J=8.0 \mathrm{~Hz}, 1 \mathrm{H}), 7.39-7.33(\mathrm{~m}, 2 \mathrm{H}), 7.09(\mathrm{dd}, J=$ 8.0, $1.2 \mathrm{~Hz}, 1 \mathrm{H}), 7.00(\mathrm{ddd}, J=8.0,8.0,1.2 \mathrm{~Hz}, 1 \mathrm{H}) \mathrm{ppm} .{ }^{13} \mathrm{C}\left\{{ }^{1} \mathrm{H}\right\}$ NMR $\left(100 \mathrm{MHz}\right.$, acetone- $\left.d_{6}\right): \delta=166.2,166.0,154.7,152.9$, 137.8, 134.7, 131.6, 131.0, 130.4, 125.6, 120.7, 119.8, 119.0, 117.6 ppm. MS (EI): $m / z=248\left(\mathrm{M}^{+}, 100\right), 204$ (85), 104 (30) 76 (84), 50 (31). The spectral data match those previously reported. ${ }^{44}$

2-(2-Hydroxyphenyl)hexahydro-1H-isoindole-1,3(2H)-dione (2v). Colorless solid, yield $=91 \%, 66.9 \mathrm{mg}$. Mp: $232-235{ }^{\circ} \mathrm{C} .{ }^{1} \mathrm{H}$ NMR (400 MHz, acetone- $d_{6}$ ): $\delta=8.62$ (br, $\left.1 \mathrm{H}\right), 7.25$ (ddd, $J=8.0$, $8.0,1.6 \mathrm{~Hz}, 1 \mathrm{H}), 7.09(\mathrm{dd}, J=8.0,1.6 \mathrm{~Hz}, 1 \mathrm{H}), 6.99(\mathrm{~d}, J=8.0 \mathrm{~Hz}$, $1 \mathrm{H}), 6.91(\mathrm{dd}, J=7.6,7.6 \mathrm{~Hz}, 1 \mathrm{H}), 3.13-3.07(\mathrm{~m}, 2 \mathrm{H}), 1.91-1.86$ $(\mathrm{m}, 4 \mathrm{H}), 1.50-1.47(\mathrm{~m}, 4 \mathrm{H}) \mathrm{ppm} .{ }^{13} \mathrm{C}\left\{{ }^{1} \mathrm{H}\right\}$ NMR $(100 \mathrm{MHz}$, acetone- $\left.d_{6}\right): \delta=179.1,154.2,130.8,130.5,121.5,120.5,117.3,41.0$, 24.6, 22.6 ppm. HRMS (ESI) calcd. for $[\mathrm{M}+\mathrm{Na}]^{+} \mathrm{C}_{14} \mathrm{H}_{15} \mathrm{NO}_{3} \mathrm{Na}$ 268.0944, found 268.0947 (1 ppm).

1-(2-Hydroxyphenyl)pyrrolidine-2,5-dione (2w). Yellow solid, yield $=89 \%, 33.9 \mathrm{mg} .{ }^{1} \mathrm{H}$ NMR $\left(400 \mathrm{MHz}\right.$, acetone- $\left.d_{6}\right): \delta=8.47$ (br, 1H), 7.28-7.24 (m, 1H), 7.08 (dd, $J=8.0,1.6 \mathrm{~Hz}, 1 \mathrm{H}), 6.98$ (dd, $J=8.4,1.2 \mathrm{~Hz}, 1 \mathrm{H}), 6.91(\mathrm{ddd}, J=7.6,7.6,1.2 \mathrm{~Hz}, 1 \mathrm{H}), 2.84$ (s, 4H) ppm. ${ }^{13} \mathrm{C}\left\{{ }^{1} \mathrm{H}\right\}$ NMR (100 MHz, acetone- $\left.d_{6}\right): \delta=177.2$, $154.2,130.9,130.5,121.3,120.5,117.5,29.4 \mathrm{ppm}$. MS (EI): $\mathrm{m} / \mathrm{z}=$ $191\left(\mathrm{M}^{+}, 100\right), 146$ (88), 136 (54), 109 (100), 55 (84). The spectral data match those previously reported. ${ }^{45}$

1-(2-Hydroxyphenyl)-3-methyl-1H-pyrrole-2,5-dione (2x). Colorless solid, yield $=89 \%, 54.2 \mathrm{mg} .{ }^{1} \mathrm{H}$ NMR $(400 \mathrm{MHz}$, acetone- $\left.d_{6}\right): \delta=8.61(\mathrm{br}, 1 \mathrm{H}), 7.28$ (ddd, $\left.J=8.0,8.0,2.0 \mathrm{~Hz}, 1 \mathrm{H}\right)$, $7.15(\mathrm{dd}, J=8.0,2.0 \mathrm{~Hz}, 1 \mathrm{H}), 7.00(\mathrm{dd}, J=8.0,1.2 \mathrm{~Hz}, 1 \mathrm{H}), 6.92$ (ddd, $J=7.6,7.6,1.2 \mathrm{~Hz}, 1 \mathrm{H}), 6.62$ (q, $J=2.0 \mathrm{~Hz}, 1 \mathrm{H}), 2.11$ (d, $J$ $=2.0 \mathrm{~Hz}, 3 \mathrm{H}) \mathrm{ppm} .{ }^{13} \mathrm{C}\left\{{ }^{1} \mathrm{H}\right\} \mathrm{NMR}\left(100 \mathrm{MHz}\right.$, acetone- $\left.d_{6}\right): \delta=$ 171.5, 170.4, 154.9, 147.0, 131.2, 130.9, 128.7, 120.52, 120.47, $117.4,11.0 \mathrm{ppm}$. MS (EI): $m / z=203\left(\mathrm{M}^{+}, 94\right), 159(100), 133(44)$, 68 (80), 52 (40). The spectral data match those previously reported..$^{36}$

2-(2-Hydroxyphenyl)-1H-benzo[de] isoquinoline-1,3(2H)-dione $(2 y)$. Colorless solid, yield $=85 \%, 59.8 \mathrm{mg} .{ }^{1} \mathrm{H}$ NMR $(400$ MHz, DMSO-d6): $\delta=9.62$ (br, $1 \mathrm{H}), 8.50$ (d, $J=7.6 \mathrm{~Hz}, 4 \mathrm{H}), 7.90$ 
(dd, $J=7.6,7.6 \mathrm{~Hz}, 2 \mathrm{H}), 7.29(\mathrm{ddd}, J=7.6,7.6,1.6 \mathrm{~Hz}, 1 \mathrm{H}), 7.24$ $(\mathrm{dd}, J=8.0,1.6 \mathrm{~Hz}, 1 \mathrm{H}), 6.99(\mathrm{dd}, J=8.0,1.6 \mathrm{~Hz}, 1 \mathrm{H}), 6.92$ (ddd, $J=7.6,7.6,1.6 \mathrm{~Hz}, 1 \mathrm{H}) \mathrm{ppm} .{ }^{13} \mathrm{C}\left\{{ }^{1} \mathrm{H}\right\}$ NMR $(100 \mathrm{MHz}$, DMSO$\left.d_{6}\right): \delta=163.3,153.4,134.3,131.4,130.6,130.3,129.5,127.9$, 127.1, 122.9, 122.7, 119.0, 116.4 ppm. MS (EI): $m / z=289\left(\mathrm{M}^{+}\right.$, 72), 272 (74), 244 (100), 126 (66). The spectral data match those previously reported. ${ }^{46}$

2-(2-Hydroxyphenyl)isoindolin-1-one (3). Colorless solid, yield $=81 \%, 54.6 \mathrm{mg} .{ }^{1} \mathrm{H}$ NMR $\left(400 \mathrm{MHz}\right.$, acetone- $\left.d_{6}\right): \delta=9.00$ (br, 1H), 7.28 (ddd, $J=7.6,1.2,1.2 \mathrm{~Hz}, 1 \mathrm{H}), 7.70-7.68(\mathrm{~m}, 2 \mathrm{H})$, $7.60-7.56(\mathrm{~m}, 1 \mathrm{H}), 7.46(\mathrm{dd}, J=8.0,1.6 \mathrm{~Hz}, 1 \mathrm{H}), 7.22$ (ddd, $J=$ 7.6, 7.6, $1.6 \mathrm{~Hz}, 1 \mathrm{H}), 7.03(\mathrm{dd}, J=8.0,1.6 \mathrm{~Hz}, 1 \mathrm{H}), 6.98(\mathrm{ddd}, J=$ 8.0, 8.0, $1.6 \mathrm{~Hz}, 1 \mathrm{H}), 5.08(\mathrm{~s}, 2 \mathrm{H}) \mathrm{ppm} .{ }^{13} \mathrm{C}\left\{{ }^{1} \mathrm{H}\right\} \mathrm{NMR}(100 \mathrm{MHz}$, acetone- $\left.d_{6}\right): \delta=169.2,152.5,143.8,133.1,132.8,129.1,128.8$, 128.0, 125.9, 124.3, 124.1, 121.2, 120.0, 53.2 ppm. MS (EI): $\mathrm{m} / \mathrm{z}=$ $225\left(\mathrm{M}^{+}, 84\right), 196$ (30), 132 (100), 120 (45). The spectral data match those previously reported. ${ }^{47}$

2-(2,6-Dihydroxyphenyl)isoindolin-1-one (4). Colorless solid, yield $=82 \%, 39.5 \mathrm{mg} . \mathrm{Mp}>250{ }^{\circ} \mathrm{C} \mathrm{dec} .{ }^{1} \mathrm{H}$ NMR $(400 \mathrm{MHz}$, acetone- $\left.d_{6}\right): \delta=8.46(\mathrm{br}, 2 \mathrm{H}), 7.79(\mathrm{~d}, J=7.6 \mathrm{~Hz}, 1 \mathrm{H}), 7.65-7.62(\mathrm{~m}$, $2 \mathrm{H}), 7.55-7,51(\mathrm{~m}, 1 \mathrm{H}), 7.05(\mathrm{dd}, J=8.4,8.4 \mathrm{~Hz}, 1 \mathrm{H}), 6.53(\mathrm{~d}, J=$ $8.4 \mathrm{~Hz}, 2 \mathrm{H}), 4.83(\mathrm{~s}, 2 \mathrm{H}) \mathrm{ppm} .{ }^{13} \mathrm{C}\left\{{ }^{1} \mathrm{H}\right\} \mathrm{NMR}(100 \mathrm{MHz}$, acetone$\left.d_{6}\right): \delta=169.3,155.8,144.4,133.6,132.4,129.7,128.5,124.15$, 124.10, 114.8, 108.8, 51.9 ppm. HRMS (ESI) calcd. for $[\mathrm{M}+\mathrm{Na}]^{+}$ $\mathrm{C}_{14} \mathrm{H} 11 \mathrm{NO} 3 \mathrm{Na} 264.0631$, found $264.0632(0 \mathrm{ppm})$.

Scale-up experiment: $\left[\mathrm{RuCl}_{2}(p \text {-cymene })\right]_{2}(0.02 \mathrm{mmol}, 12.2$ $\mathrm{mg}, 0.01$ eq.), ammonium persulfate ( $2.4 \mathrm{mmol}, 548 \mathrm{mg}, 1.2 \mathrm{eq}$.), substrate 1a ( $2 \mathrm{mmol}, 1$ eq.) and TFA/TFAA ( $6 \mathrm{~mL}, 3: 1, v / v)$ were introduced in a flame-dried Schlenk tube under air atmosphere. The reaction mixture was stirred at $80{ }^{\circ} \mathrm{C}$ during 15 hours. Then, the reaction mixture was cooled down to room temperature and diluted with $\mathrm{H}_{2} \mathrm{O}(200 \mathrm{~mL})$ followed by extraction with $\mathrm{CH}_{2} \mathrm{Cl}_{2}(3 \times 100$ $\mathrm{mL})$. The combined organic layers were washed with brine $(200$ $\mathrm{mL}$ ) and dried over $\mathrm{Na}_{2} \mathrm{SO}_{4}$. After filtration and evaporation of the solvents in vacuo, the crude product was purified by column chromatography on silica gel ( $n$-heptane/EtOAc: $4 / 1, v / v)$ to give product $2 \mathbf{a}$ as a solid in $82 \%$ yield (396 $\mathrm{mg}$ ).

Synthesis and characterization of $\mathbf{5}$. A solution of $\mathbf{2 a}(0.4$ mmol, 1 eq.) and $\mathrm{K}_{2} \mathrm{CO}_{3}(0.6 \mathrm{mmol}, 1.5$ eq. $)$ in $\mathrm{DMF}(2 \mathrm{~mL})$ was stirred at room temperature, then iodomethane $(0.6 \mathrm{mmol}, 1.5 \mathrm{eq}$. was added. After $2 \mathrm{~h}$, the reaction mixture was diluted with $\mathrm{H}_{2} \mathrm{O}$ $(40 \mathrm{~mL})$ and extracted with ethyl acetate $(3 \times 20 \mathrm{~mL})$. The combined organic phase was washed with brine, dried over anhydrous $\mathrm{Na}_{2} \mathrm{SO}_{4}$ and concentrated in vacuo. The crude product was purified by column chromatography ( $n$-heptane/EtOAc: $5 / 1, v / v$ ) to obtain product $\mathbf{5}$ in $96 \%$ yield as a white solid.

$N$-o-Methoxyphenylphthalimide (5). Colorless solid, yield $=$ $96 \%, 48.6$ mg. ${ }^{1} \mathrm{H}$ NMR $\left(400 \mathrm{MHz}, \mathrm{CDCl}_{3}\right): \delta=7.94$ (dd, $J=5.6$, $3.2 \mathrm{~Hz}, 2 \mathrm{H}), 7.77(\mathrm{dd}, J=5.6,3.2 \mathrm{~Hz}, 2 \mathrm{H}), 7.46-7.42(\mathrm{~m}, 1 \mathrm{H}), 7.27-$ $7.25(\mathrm{~m}, 1 \mathrm{H}), 7.10-7.04(\mathrm{~m}, 2 \mathrm{H}), 3.80(\mathrm{~s}, 3 \mathrm{H}) \mathrm{ppm} .{ }^{13} \mathrm{C}\left\{{ }^{1} \mathrm{H}\right\} \mathrm{NMR}$ $\left(100 \mathrm{MHz}, \mathrm{CDCl}_{3}\right): \delta=167.3,155.4,134.1,132.2,130.6,130.0$, $123.6,120.8,120.2,112.1,55.8 \mathrm{ppm}$. The spectral data match those previously reported. ${ }^{24}$

Synthesis and characterization of $6 .\left[\mathrm{RuCl}_{2}(p \text {-cymene })\right]_{2}$ ( $0.002 \mathrm{mmol}, 1.2 \mathrm{mg}, 0.01$ eq.), potassium carbonate ( $0.6 \mathrm{mmol}$, $82.9 \mathrm{mg}, 3$ eq.), distilled water ( $0.3 \mathrm{mmol}, 5.4 \mathrm{mg}, 5.4 \mu \mathrm{L}, 1.5$ eq. $)$, substrate $1 \mathrm{a}(0.2 \mathrm{mmol}, 1$ eq.) and $N$-methyl-2-pyrrolidine $(1.0 \mathrm{~mL})$ were introduced in a flame-dried Schlenk tube under argon atmosphere. The reaction mixture was stirred at $150{ }^{\circ} \mathrm{C}$ during 6 hours. Then, the reaction mixture was cooled down to room temperature and diluted with water $(10 \mathrm{~mL})$ followed by addition of $\mathrm{HCl}(1.0$ M) until $\mathrm{pH}$ reached 7. The aqueous phase was extracted with ethyl acetate and the combined organic phases were dried over anhydrous $\mathrm{MgSO}_{4}$, filtered, and concentrated in vacuo. After solvents evaporation under vacuum, product 6 was purified by column chromatography (petroleum ether/EtOAc: $5 / 1, v / v$ ) in $78 \%$ yield.
$N$-(o-Methoxyphenyl)benzamide (6). Colorless solid, yield $=$ $78 \%, 70.9 \mathrm{mg} .{ }^{1} \mathrm{H}$ NMR $\left(400 \mathrm{MHz}, \mathrm{CDCl}_{3}\right): \delta=8.55(\mathrm{dd}, J=7.6$, $1.6 \mathrm{~Hz}, 2 \mathrm{H}), 7.90(\mathrm{dd}, J=6.8,1.6 \mathrm{~Hz}, 2 \mathrm{H}), 7.57-7.48(\mathrm{~m}, 3 \mathrm{H}), 7.11-$ $7.01(\mathrm{~m}, 2 \mathrm{H}), 6.93(\mathrm{dd}, J=8.0,1.2 \mathrm{~Hz}, 1 \mathrm{H}), 3.93$ (s, 3H) ppm. ${ }^{13} \mathrm{C}\left\{{ }^{1} \mathrm{H}\right\}$ NMR $\left(100 \mathrm{MHz}, \mathrm{CDCl}_{3}\right): \delta=165.2,148.1,135.3,131.6$, $128.7,127.8,127.0,123.8,121.2,119.8,109.9,55.8$ ppm. MS (EI): $m / z=227\left(\mathbf{M}^{+}, 29\right), 105(100), 77$ (46). The spectral data match those previously reported. ${ }^{48}$

Synthesis and characterization of 7. A solution of $\mathbf{5}(0.2$ mmol) and $\mathrm{NH}_{2} \mathrm{NH}_{2} \cdot \mathrm{H}_{2} \mathrm{O}(80 \mu \mathrm{L})$ in ethanol $(1 \mathrm{~mL})$ was heated under reflux for $2 \mathrm{~h}$. The reaction mixture was cooled to room temperature, and the mixture was filtered through Celite. The solvent was removed, and the crude product was purified using column chromatography $(n$-heptane/EtOAc: $10 / 1, v / v)$ to give a red oil 7 in $83 \%$ yield.

o-Anisidine (7). Red oil, yield $=83 \%, 20.4 \mathrm{mg} .{ }^{1} \mathrm{H}$ NMR $(400$ MHz, acetone- $\left.d_{6}\right): \delta=6.79(\mathrm{~d}, J=7.6 \mathrm{~Hz}, 1 \mathrm{H}), 6.69(\mathrm{dd}, J=5.2$, $1.2 \mathrm{~Hz}, 2 \mathrm{H}), 6.57$ (ddd, $J=8.0,4.8,4.8 \mathrm{~Hz}, 1 \mathrm{H}), 4.32$ (br, $2 \mathrm{H}), 3.80$ (s, 3H) ppm. ${ }^{13} \mathrm{C}\left\{{ }^{1} \mathrm{H}\right\}$ NMR $\left(100 \mathrm{MHz}\right.$, acetone- $\left.d_{6}\right): \delta=147.9$, $138.4,121.8,117.8,115.0,111.3,55.7 \mathrm{ppm}$. The spectral data match those previously reported. ${ }^{49}$

\section{ASSOCIATED CONTENT}

\section{Supporting Information.}

The Supporting Information is available free of charge on the ACS Publications website at DOI: XXX.

Screening of reaction conditions, computational details and NMR spectra (PDF)

Crystallographic data for $\mathbf{2 b}$ (CCDC-187307, CIF)

Crystallographic data for 2d (CCDC-1873708, CIF)

\section{AUTHOR INFORMATION}

\section{Corresponding Author}

*E-mail: rafael.gramage-doria@univ-rennes1.fr

\section{ORCID}

Christian Bruneau: 0000-0002-2220-1458

Rafael Gramage-Doria: 0000-0002-0961-4530

Notes

The authors declare no competing financial interest

\section{ACKNOWLEDGMENTS}

Financial support by CNRS, Universite de Rennes 1, Rennes Metropole, China Scholarship Council (Ph.D. grant to Y.-C.Y.), and COST Action CA15106 (CHAOS) is acknowledged.

\section{REFERENCES}

(1) (a) Chen, M. S.; White, M. C. A Predictably Selective Aliphatic C-H Oxidation Reaction for Complex Molecule Synthesis. Science 2007, 318, 783. (b) Fuchs, P. L. Handbook of Reagents for Organic Synthesis: Catalytic Oxidation Reactions, Wiley-VCH: Weinheim, 2013. (c) Stahl, S. S.; Alsters, P. L. Liquid Phase Aerobic Oxidation Catalysis: Industrial Applications and Academic Perspectives, Wiley-VCH: Weinheim, 2016. (d) Muniz, K. Science of Synthesis: Catalytic Oxidation in Organic Synthesis, Thieme: Germany, 2018.

(2) Mizuno, N. Modern Heterogeneous Oxidation Catalysis: Design, Reactions and Characterization, Wiley-VCH: Weinheim, 2009.

(3) (a) Newhouse, T.; Baran, P. S. If C-H Bonds Could Talk: Selective C-H Bond Oxidation. Angew. Chem. Int. Ed. 2011, 50, 3362. (b) Boisvert, L.; Goldberg, K. I. Reactions of Late Transition Metal Complexes with Molecular Oxygen. Acc. Chem. Res. 2012, 45, 899. (c) Qiu, Y.; Gao, S. Trends in Applying C-H Oxidation to the Total Synthesis of Natural Products. Nat Prod. Rep. 2016, 33, 562. (d) Liang, Y.-F.; Jiao, N. Oxygenation via C-H /C-C Bond Activation with Molecular Oxygen. Acc. Chem. Res. 2017, 50, 1640. (e) Dong, J.; Fernandez-Fueyo, E.; Hollmann, F.; Paul, C. E.; Pesic, 
M. Schmidt, S.; Wang, Y.; Younes, S.; Zhang, W. Biocatalytic Oxidation Reactions: A Chemist's Perspective. Angew. Chem. Int. Ed. 2018, 57, 9238.

(4) (a) Ray, K.; Pfaff, F. F.; Wang, B.; Nam, W. Status of Reactive NonHeme Metal-Oxygen Intermediates in Chemical and Enzymatic Reactions. J. Am. Chem. Soc. 2014, 136, 13942. (b) McCann, S. D.; Stahl, S. S. Copper-Catalyzed Aerobic Oxidations of Organic Molecules: Pathways for Two-Electron Oxidation with a Four-Electron Oxidant and a One-Electron Redox-Active Catalyst. Acc. Chem. Res. 2015, 48, 1756. (c) Gagnon, N.; Tolman, W. B. $[\mathrm{CuO}]^{+}$and $[\mathrm{CuOH}]^{2+}$ Complexes: Intermediates in Oxidation Catalysis? Acc. Chem. Res. 2015, 48, 2126. (d) Itoh, S. Developing Mononuclear Copper-Active-Oxygen Complexes Relevant to Reactive Intermediates of Biological Oxidation Reactions. Acc. Chem. Res. 2015, 48, 2066. (e) Oloo, W. N.; Que Jr., L. Bioinspired Nonheme Iron Catalysts for $\mathrm{C}-\mathrm{H}$ and $\mathrm{C}=\mathrm{C}$ Bond Oxidation: Insights into the Nature of the Metal-Based Oxidants. Acc. Chem. Res. 2015, 48, 2612. (f) Lindhorst, A. C.; Haslinger, S.; Kuhn, F. E. Molecular Iron Complexes as Catalysts for Selective C-H Bond Oxygenation Reactions. Chem. Commun. 2015, 51, 17193. (g) Engelmann, X.; Monte-Perez, I.; Ray, K. Oxidation Reactions with Bioinspired Mononuclear Non-Heme Metal-Oxo Complexes. Angew. Chem. Int. Ed. 2016, 55, 7632. (h) Olivo, G.; Cusso, O. Costas, M. Biologically Inspired $\mathrm{C}-\mathrm{H}$ and $\mathrm{C}=\mathrm{C}$ Oxidations with Hydrogen Peroxide Catalyzed by Iron Coordination Complexes. Chem. Asian J. 2016, 11, 3148. (i) Jasniewski, A. J.; Que Jr., L. Dioxygen Activation by Nonheme Diiron Enzymes: Diverse Dioxygen Adducts, High-Valent Intermediates, and Related Model Complexes. Chem. Rev. 2018, 118, 2554.

(5) (a) Alonso, D. A.; Najera, C.; Pastor, I.M.; Yus, M. Transition-MetalCatalyzed Synthesis of Hydroxylated Arenes. Chem. Eur. J. 2010, 16, 5274 (b) Lyons, T. W.; Sanford, M. S. Palladium-Catalyzed Ligand-Directed CH Functionalization Reactions. Chem. Rev. 2010, 110, 1147. (c) Enthaler, S.; Company, A. Palladium-Catalyzed Hydroxylation and Alkoxylation. Chem. Soc. Rev. 2011, 40, 4912. (d) Rao, Y. Pd-Catalyzed sp ${ }^{2}$ C-H Hydroxylation with TFA/TFAA via Weak coordinations. Synlett 2013, 24, 2472. (e) Thirunavukkarasu, V. S.; Kozhushkov, S. I.; Ackermann, L. C-H Nitrogenation and Oxygenation by Ruthenium Catalysis. Chem. Commun. 2014, $50,29$.

(6) (a) Yang, X.; Sun, Y.; Chen, Z.; Rao, Y. A General Approach Towards Catechol and Pyrogallol Through Ruthenium- and Palladium-Catalyzed C-H Hydroxylation by Weak Coordination. Adv. Synth. Catal. 2014, 356, 1625. (b) Yang, F.; Rauch, K.; Kettelhoit, K. Ackermann, L. Aldehyde-Assisted Ruthenium(II)-Catalyzed C-H Oxygenations. Angew. Chem. Int. Ed. 2014, 53, 11285. (c) Kim, K; Choe, H.; Jeong, Y.; Lee, J. H.; Hong, S. Ru(II)-Catalyzed Site-Selective Hydroxylation of Flavone and Chromone Derivatives: The Importance of the 5-Hydroxyl Motif for the Inhibition of Aurora Kinases. Org. Lett. 2015, 17, 2550. (d) Li, Y.-Q.; Yang, Q.L.; Fang, P.; Mei, T.-S.; Zhang, D. Palladium-Catalyzed C( $\left.\mathrm{sp}^{2}\right)-\mathrm{H}$ Acetoxylation via Electrochemical Oxidation. Org. Lett. 2017, 19, 2905. (e) Guo, Y.; Yu, K.-K.; Xing, L.-H.; Liu, H.-W.; Wang, W.; Ji, Y.-F. PalladiumCatalyzed Divergent Regioselective Homocoupling and Hydroxylation of 3-Arylbenzo $[d]$ isoxazoles. Adv. Synth. Catal. 2017, 359, 410. (f) Raghuvanshi, K.; Zell, D.; Ackermann, L. Ruthenium(II)-Catalyzed C-H Oxygenations of Reusable Sulfoximine Benzamides. Org. Lett. 2017, 19, 1278. (g) Dias, G. G.; Rogge, T.; Kuniyil, R.; Jacob, C.; Menna-Barreto, R. F. S.; da Silva Junior, E. N.; Ackermann, L. Ruthenium-Catalyzed C-H Oxygenation of Quinones by Weak O-Coordination for Potent Trypanocidal Agents. Chem. Commun. 2018, 54, 12840 and references cited therein.

(7) (a) Thirunavukkarasu, V. S.; Ackermann, L. Ruthenium-Catalyzed $\mathrm{C}-\mathrm{H}$ Bond Oxygenations with Weakly Coordinating Ketones. Org. Lett. 2012, 14, 6206. (b) Shan, G.; Yang, X.; Ma, L.; Rao, Y. Pd-Catalyzed C-H Oxygenation with TFA/TFAA: Expedient Access to Oxygen-Containing Heterocycles and Late-Stage Drug Modification. Angew. Chem. Int. Ed. 2012, 51, 13070. (c) Mo, F.; Trzepkowski, L. J.; Dong, G. Synthesis of ortho-Acylphenols through the Palladium-Catalyzed Ketone-Directed Hydroxylation of Arenes. Angew. Chem. Int. Ed. 2012, 51, 13075. (d) Choy, P. Y.; Kwong, F. Y. Palladium-Catalyzed ortho-CH-Bond Oxygenation of Aromatic Ketones. Org. Lett. 2013, 15, 270. (e) Yang, X.; Shan, G.; Rao, Y. Synthesis of 2-Aminophenols and Heterocycles by Ru-Catalyzed C-H Mono- and Dihydroxylation. Org. Lett. 2013, 15, 2334. (f) Zhang, H.-Y.; Yi, H.-M. Wang, G.-W.; Yang, B.; Yang, S.-D. Pd(II)-Catalyzed C( $\left.\mathrm{sp}^{2}\right)-\mathrm{H}$ Hydroxylation with $\mathrm{R}_{2}(\mathrm{O}) \mathrm{P}-\mathrm{Coordinating}$ Group. Org. Lett. 2013, 15, 6186. (g) Shan, G.; Han, X.; Lin, Y.; Yu, S.; Rao, Y. Broadening the Catalyst and Reaction Scope of Regio- and Chemoselective C-H Oxygenation: a Convenient and Scalable Approach to 2-Acylphenols by Intriguing $\mathrm{Rh}(\mathrm{II})$ and $\mathrm{Ru}$ (II) catalysis. Org. Biomol. Chem. 2013, 11, 2318.
(8) Sun, Y.-H.; Sun, T.-Y.; Wu, Y.-D.; Zhang, X.; Rao, Y. A DiversityOriented Synthesis of Bioactive Benzanilides via a Regioselective C( $\left.\mathrm{sp}^{2}\right)$ H Hydroxylation Strategy. Chem. Sci. 2016, 7, 2229.

(9) (a) Chen, T.-A.; Jen, A. K.-Y.; Cai, Y. Facile Approach to Nonlinear Optical Side-Chain Aromatic Polyimides with Large Second-Order Nonlinearity and Thermal Stability. J. Am. Chem. Soc. 1995, 117, 7295. (b) Yu, D.; Ghavari, A.; Yu, L. Novel Aromatic Polyimides for Nonlinear Optics. J. Am. Chem. Soc. 1995, 117, 11680. (c) Han, S. H.; Misdan, N.; Kim, S.; Doherty, C. M.; Hill, A. J.; Lee, Y. M. Thermally Rearranged (TR) Polybenzoxazole: Effects of Diverse Imidization Routes on Physical Properties and Gas Transport Behaviors. Macromolecules 2010, 43, 7657.

(10) (a) Odell, L. R.; Howan, D.; Gordon, C. P.; Robertson, M. J.; Chau, N.; Mariana, A.; Whiting, A. E.; Abagyan, R.; Daniel, J. A.; Gorgani, N. N.; Robinson, P. J.; McCluskey, A. The Pthaladyns: GTP Competitive Inhibitors of Dynamin I and II GTPase Derived from Virtual Screening. $J$. Med.Chem. 2010, 53, 5267. (b) Mangani, S.; Cancian, L.; Leone, R.; Pozzi, C.; Lazzari, S.; Luciani, R.; Ferrari, S.; Costi, M. P. Identification of the Binding Modes of N-Phenylphthalimides Inhibiting Bacterial Thymidylate Synthase through X-Ray Crystallography Screening. J. Med. Chem. 2011, $54,5454$.

(11) (a) Engle, K. M.; Mei, T.-S.; Wasa, M.; Yu, J.-Q. Weak Coordination as a Powerful Means for Developing Broadly Useful C-H Functionalization Reactions. Acc. Chem. Res. 2012, 45, 788. (b) de Sarkar, S.; Liu, W.; Kozhushkov, S. I.; Ackermann, L. Weakly Coordinating Directing Groups for Ruthenium(II)-Catalyzed C-H Activation. Adv. Synth. Catal. 2014, 356, 1461.

(12) (a) Nakazono, S.; Imazaki, Y.; Yoo, H.; Yang, J.; Sasamori, T.; Tokitoh, N.; Cedric, T.; Kageyama, H.; Kim, D.; Shinokubo, H.; Osuka, A. Regioselective Ru-Catalyzed Direct 2,5,8,11-Alkylation of Perylene Bisimides. Chem. Eur. J. 2009, 15, 7530. (b) Nakazono, S.; Easwaramoorthi, S.; Kim, D. Shinokubo, H.; Osuka, A. Synthesis of Arylated Perylene Bisimides Through C-H Bond Cleavage Under Ruthenium Catalysis. Org. Lett. 2009, 11, 5426. (c) Sharma, S.; Khan, I. A.; Saxena, A. K. Room Temperature Palladium-Catalyzed Decarboxylative Acyl/Aroylation using [Fe(III) $($ EDTA $\left.)\left(\eta^{2}-\mathrm{O}_{2}\right)\right]^{3-}$ as Oxidant at Biological pH. Adv. Synth. Catal. 2013, 355, 673. (d) Leitch, J. A.; Cook, H. P.; Bhonoah, Y.; Frost, C. G. Use of the Hydantoin Directing Group in Ruthenium(II)-Catalyzed C-H Functionalization. J. Org. Chem. 2016, 81, 10081. (e) Zhang, L.; He, D.; Liu, Y.; Wang, K.; Guo, Z.; Lin, J.; Zhang, H.-J. 2,5,8,11-Tetraalkenyl Perylene Bisimides: Direct Regioselective Synthesis and Enhanced $\pi-\pi$ Stacking Interaction $\mathrm{Org}$. Lett. 2016, 18, 5908. (f) Hameury, S.; Kunz, S.; Sommer, M. Expanding the Scope of Electron-Deficient C-H Building Blocks: Direct Arylation of Pyromellitic Acid Diimide. ACS Omega 2017, 2, 2483. (g) Zhang, C.; Song, Y.; Sang, Z.; Zhan, L. Rao, Y. Mixing O-Containing and N-Containing Directing Groups for C-H Activation: A Strategy for the Synthesis of Highly Functionalized 2,2'-Biaryls. J. Org. Chem. 2018, 83, 2582.

(13) (a) Raghuvanshi, K.; Rauch, K.; Ackermann, L. Ruthenium(II)Catalyzed C-H Acyloxylation of Phenols with Removable Auxiliary. Chem. Eur. J. 2015, 21, 1790. (b) Sarkar, T.; Pradhan, S.; Punniyamurthy, T. Ruthenium(II)-Catalyzed Positional Selective C-H Oxygenation of N-Aryl-2pyrimidines. J. Org. Chem. 2018, 83, 6444.

(14) (a) Wu, Y.; Zhu, W. Organic Sensitizers from D- $\pi$-A to D-A- $\pi$-A: Effect of the Internal Electron-Withdrawing Units on Molecular Absorption, Energy Levels and Photovoltaic Performances. Chem. Soc. Rev. 2013, 42, 2039. (b) Hendsbee, A. D.; Sun, J.-P.; Law, W. K.; Yan, H.; Hill, I. G.; Spasyuk, D. M.; Welch, G. C. Synthesis, Self-Assembly, and Solar Cell Performance of N-Annulated Perylene Diimide Non-Fullerene Acceptors. Chem. Mater. 2016, 28, 7098.

(15) Tullos, G. L.; Powers, J. M.; Jeskey, S. J.; Mathias, L. J. Thermal Conversion of Hydroxy-Containing Imides to Benzoxazoles: Polymer and Model Compound Study. Macromolecules 1999, 32, 3598.

(16) (a) Yuan, Y.-C.; Kamaraj, R.; Bruneau, C.; Labasque, T.; Roisnel, Gramage-Doria, R. Unmasking Amides: Ruthenium-Catalyzed Protodecarbonylation of N-Substituted Phthalimide Derivatives. Org. Lett. 2017, 19, 6404. (b) Yuan, Y.-C; Bruneau, C.; Gramage-Doria, R. Merging Transition Metal Catalysis with Phthalimides: a New Entry to Useful Building Blocks. Synthesis 2018, 50, 4216.

(17) Hsieh, J.-C.; Cheng, C.-H. Nickel-Catalyzed Coupling of Isocyanates with 1,3-Iodo Esters and Halobenzenes: a Novel Method for the Synthesis of Imide and Amide Derivatives. Chem. Commun. 2005, 36, 4554.

(18) Zakharova, N. A.; Khromov-Borisov, N. V. Alkylated Aromatic Amines. VIII. Role of the Spatial Factor in the Reactions of Methyl Iodide with Monotertiary p-Phenylenediamines Zhurnal Organicheskoi Khimii, 1970, 6, 116. 
(19) Rasmussen, L. K.; Begtrup, M.; Ruhland, T. Resin-Bound Triaryl Bismuthanes and Bismuth Diacetates: Novel Multidirectional Linkers and Novel Resin-Bound Arylation Reagents. J. Org. Chem. 2004, 69, 6890.

(20) Wang, X.; Xiong, W.; Huang, Y.; Zhu, J.; Hu, Q.; Wu, W.; Jiang, H. Palladium-Catalyzed Synthesis of $1 \mathrm{H}$-Indenes and Phthalimides via Isocyanide Insertion. Org. Lett. 2017, 19, 5818.

(21) Shrestha, R.; Mukherjee, P.; Tan, Y.; Litman, Z. C.; Hartwig, J. F. Sterically Controlled, Palladium-Catalyzed Intermolecular Amination of Arenes. J. Am. Chem. Soc. 2013, 135, 8480.

(22) Worlikar, S. A.; Larock, R. C. Palladium-Catalyzed One-Step Synthesis of Isoindole-1,3-diones by Carbonylative Cyclization of $o$-Halobenzoates and Primary Amines J. Org. Chem. 2008, 73, 7175.

(23) Kumar, V.; Banker, G. S. Incompatibility of Polyvinyl Acetate Phthalate with Benzocaine: Isolation and Characterization of 4Phthalimidobenzoic Acid Ethyl Ester. Int. J. Pharm. 1992, 79, 61.

(24) Sharma, N.; Sekar, G. Stable and Reusable Binaphthyl-Supported Palladium Catalyst for Aminocarbonylation of Aryl Iodides. Adv. Synth. Catal. 2016, 358, 314

(25) Yedage, S. L.; D'silva, D. S.; Bhanage, B. M. $\mathrm{MnO}_{2}$ Catalyzed Formylation of Amines and Transamidation of Amides Under Solvent-Free Conditions. $R S C A d v . \mathbf{2 0 1 5}, 5,80441$.

(26) Liu, S.; Deng, Q.; Fang, W.; Gong, J.-F.; Song, M.-P.; Xua, M.; Tu, T. Efficient and Scalable Pd-Catalyzed Double Aminocarbonylations Under Atmospheric Pressure at Low Catalyst Loadings. Org. Chem. Front. 2014, 1,1261

(27) Dong, X.-F.; Fan, J.; Shi, X.-Y.; Liu, K.-Y.; Wang, P.-M.; Wei, J.F. Ruthenium(II)-Catalyzed N-Substituted Phthalimide Synthesis via C-H Activation/[3+2] Annulation. J. Organomet. Chem. 2015, 779, 55.

(28) Sarkar, S. D.; Ackermann, L. Ruthenium(II)-Catalyzed C-H Activation with Isocyanates: A Versatile Route to Phthalimides. Chem. Eur. J. 2014, 20, 13932.

(29) Williams, F. J.; Donahue, P. E. Reactions of Phenoxides with Nitroand Halo-Substituted Phthalimides. J. Org. Chem. 1977, 42, 3414.

(30) Shibata, Y.; Sasaki, K.; Y. Hashimoto, Y.; Iwasaki, S. Phenylphthalimides with Tumor Necrosis Factor Alpha Production-Enhancing Activity. Chem. Pharm. Bull. 1996, 44, 156.

(31) Wang, M.; Lu, J.; Ma, J.; Zhang, Z.; Wang, F. Cuprous Oxide Catalyzed Oxidative C-C Bond Cleavage for C-N bond formation: Synthesis of Cyclic Imides from Ketones and Amines. Angew. Chem. Int. Ed. 2015 , 54,14061

(32) Kim, H. J.; Kim, J.; Cho, S. H.; Chang, S. Intermolecular Oxidative C-N Bond Formation under Metal-Free Conditions: Control of Chemoselectivity between Aryl $\mathrm{sp}^{2}$ and Benzylic $\mathrm{sp}^{3} \mathrm{C}-\mathrm{H}$ Bond Imidation. J. Am. Chem. Soc. 2011, 133, 16382.

(33) Kaminski, K.; Wiklik, B.; Obniska, J. Synthesis, Anticonvulsant Properties, and SAR Analysis of Differently Substituted Pyrrolidine-2,5diones and Piperidine-2,6-diones. Arch. Pharm. Chem. Life Sci. 2014, 347, 840.

(34) Kobeissi, M.; Yazbeck, O.; Chreim, Y. A Convenient One-Pot Synthesis of Polysubstituted Pyrroles from N-Protected Succinimides. Tetrahedron Lett. 2014, 552523.

(35) Matuszak, N.; Muccioli, G. G.; Labar, G.; Lambert, D. M. Synthesis and in Vitro Evaluation of N-Substituted Maleimide Derivatives as Selective Monoglyceride Lipase Inhibitors. J. Med. Chem. 2009, 52, 7410.

(36) Pyriadi, T. M.; Alazawi, A. M. Synthesis, Polymerization, and Curing of N-Substituted Citraconimidyl Acrylate. J. Polym. Sci. Part A: Polym. Chem. 1999, 37, 427.

(37) (a) Kumar, V.; Sharma, S.; Sharma, U.; Singh, B.; Kumar, N. Synthesis of Substituted Amines and Isoindolinones: Catalytic Reductive Amination Using Abundantly Available $\mathrm{AlCl}_{3} / \mathrm{PMHS}$. Green Chem. 2012, 14 3410. (b) Wang, S.; Huang, H.; Bruneau, C.; Fischmeister, C. Selective and Efficient Iridium Catalyst for the Reductive Amination of Levulinic Acid into Pyrrolidones. ChemSusChem 2017, 10, 4150.

(38) Lin, C.; Zhen, L.; Cheng, Y.; Du, H.-J.; Zhao, H.; Wen, X.; Kong, L.-Y.; Xu, Q.-L.; Sun, H. Visible Light-Induced Isoindole Formation To Trigger Intermolecular Diels-Alder Reactions in the Presence of Air. Org. Lett. 2015, 17, 2684.

(39) Perry, R. J.; Turner, S. R. Preparation of N-Substituted Phthalimides by the Palladium-Catalyzed Carbonylation and Coupling of o-Dihalo Aromatics and Primary Amines. J. Org. Chem. 1991, 56, 6573.

(40) Chu, C. D.; Qi, Y. H.; Hao, W. A More Efficient Synthetic Process of N-Arylphthalimides in Ionic Liquid [bmim] $\left[\mathrm{BF}_{4}\right]$. Catal. Commun. 2007, $8,1527$.
(41) Wójcik, P.; Trzeciak, A. M. The aminocarbonylation of 1,2-diiodoarenes with primary and secondary amines catalyzed by palladium complexes with imidazole ligands. Appl. Catal. A 2018, 560, 73.

(42) Yao, C.; Bao, Y.; Lu, T.; Zhou, Q. Stereoselective Synthesis of Functionalized Benzooxazepino[5,4-a]isoindolone Derivatives via Cesium Carbonate Catalyzed Formal [5+2] Annulation of 2-(2-Hydroxyphenyl)isoindoline-1,3-dione with Allenoates. Org. Lett. 2018, 20, 2152.

(43) Yamamoto, Y.; Hisa, T.; Arai, J.; Saito, Y.; Yamamoto, F.; Mukai, T.; Ohshima, T.; Maeda, M.; Ohkubo, Y. Isomeric Methoxy Analogs of Nimesulide for Development of Brain Cyclooxygense-2 (COX-2)-Targeted Imaging Agents: Synthesis, in Vitro COX-2-Inhibitory Potency, and Cellular Transport Properties. Bioorg. Med. Chem. 2015, 23, 6807.

(44) Mansor, S.; Zakaria, N.; Ariffin, A.; Ng, S. W. N-(2-Hydroxyphenyl)-4-nitrophthalimide. Acta Cryst. 2008, E64, o1770.

(45) Caulfield, M. J.; Looney, M. G.; Pittard, R. A.; Solomon, D. H. Studies on Polyimides. Part 1: Synthesis of Model Compounds and Their Reaction with Hexamethylenetetramine. Polvmer 1998, 39, 6541.

(46) Wang, J.; Yang, L.; Hou, C.; Cao, H. A New N-Imidazolyl-1,8naphthalimide Based Fluorescence Sensor for Fluoride Detection. Org. Biomol. Chem. 2012, 10, 6271.

(47) Khadim, M. A.; Colebrook, L. D. Carbon-13 delta shifts and steric interactions in N-Aryl-1-isoindolinones and -isoindoline-1,3-diones. Magnet.. Res. Chem. 1985, 23, 259.

(48) Xu, H.; Wolf, C. Copper Catalyzed Coupling of Aryl Chlorides, Bromides and Iodides with Amines and Amides. Chem. Commun. 2009, 1715.

(49) Duan, Y.; Song, T.; Dong, X.; Yang, Y. Enhanced Catalytic Performance of Cobalt Nanoparticles Coated with a N,P-Codoped Carbon Shell Derived from Biomass for Transfer Hydrogenation of Functionalized Nitroarenes. Green Chem. 2018, 20, 2821. 
Insert Table of Contents artwork here

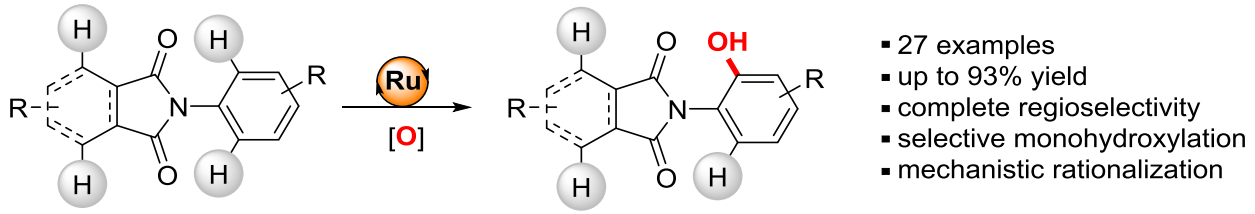

